\title{
Conservative Multigrid Methods for Cahn-Hilliard Fluids
}

\author{
Junseok Kim $^{\mathrm{a}}$, Kyungkeun Kang ${ }^{\mathrm{b}}$, and John Lowengrub ${ }^{\mathrm{c}}$ \\ ${ }^{a}$ School of Mathematics, University of Minnesota, Minneapolis, MN 55455 \\ ${ }^{\mathrm{b}}$ Max-Planck-Institute for Mathematics in the Sciences Inselstr. 22 - 26, D-04103 Leipzig, \\ Germany \\ ${ }^{c}$ School of Mathematics, University of Minnesota, Minneapolis, MN 55455
}

\begin{abstract}
We develop a conservative, second order accurate fully implicit discretization in two dimensions of the Navier-Stokes NS and Cahn-Hilliard CH system that has an associated discrete energy functional. This system provides a diffuse-interface description of binary fluid flows with compressible or incompressible flow components [44,4]. In this work, we focus on the case of flows containing two immiscible, incompressible and density-matched components. The scheme, however, has a straightforward extension to multi-component systems. To efficiently solve the discrete system at the implicit time-level, we develop a nonlinear multigrid method to solve the $\mathbf{C H}$ equation which is then coupled to a projection method that is used to solve the NS equation. We analyze and prove convergence of the scheme in the absence of flow. We demonstrate convergence of our scheme numerically in both the presence and absence of flow and perform simulations of phase separation via spinodal decomposition. We examine the separate effects of surface tension and external flow on the decomposition. We find surface tension driven flow alone increases coalescence rates through the retraction of interfaces. When there is an external shear flow, the evolution of the flow is nontrivial and the flow morphology repeats itself in time as multiple pinchoff and reconnection events occur. Eventually, the periodic motion ceases and the system relaxes to a global equilibrium. The equilibria we observe appears has a similar structure in all cases although the dynamics of the evolution is quite different. We view the work presented in this paper as preparatory for the detailed investigation of liquid/liquid interfaces with surface tension where the interfaces separate two immiscible fluids [37]. To this end, we include a simulation of the pinchoff of a liquid thread under the Rayleigh instability at finite Reynolds number.
\end{abstract}

Key words: Cahn-Hilliard equation, nonlinear multigrid method, fluid flow, interfacial tension 


\section{Introduction}

The Cahn-Hilliard equation is the prototypical continuum model of phase separation. It was originally proposed by Cahn and Hilliard [14] to model binary alloys and has subsequently been adopted to model many other physical situations such as phase transitions and interface dynamics in multiphase fluids [32] which we consider here. Phase separation occurs, for example, when a single phase homogeneous system composed of two fluid components, in thermal equilibrium (e.g. at a high temperature), is rapidly cooled to a temperature $T$ below a critical temperature $T_{c}$ where the system is unstable with respect to infinitesimal concentration fluctuations. Spinodal decomposition then takes place and the system separates into spatial regions rich in one component and poor in the other. The evolution lowers the free energy and leads to an equilibrium state with coexisting phases (e.g. see $[13,48,10,47])$. Assuming that the fluid components are incompressible $(\nabla \cdot \mathbf{u}=0)$ with equal densities (set to one for simplicity) and that the evolution is isothermal, the nondimensional Cahn-Hilliard $\mathbf{C H}$ model is as follows. Let $c$ be the phase variable (i.e. concentration), then

$$
\begin{aligned}
& c_{t}(\mathbf{x}, t)+\nabla \cdot(\mathbf{u} c)=\frac{1}{\mathbf{P e}} \nabla \cdot(M(c) \nabla \mu(\mathbf{x}, t)), \text { for }(\mathbf{x}, t) \in \Omega \times[0, T] \subset \mathbb{R}^{n} \times \mathbb{R} \\
& \text { and } \\
& \qquad \mu(\mathbf{x}, t)=\phi(c(\mathbf{x}, t))-\epsilon^{2} \Delta c(\mathbf{x}, t),
\end{aligned}
$$

where $\mathbf{u}$ is the mass-averaged fluid velocity (i.e. $\mathbf{u}=\mathbf{u}_{1}+\mathbf{u}_{2}$, where $\mathbf{u}_{1}$ and $\mathbf{u}_{2}$ are the velocities of the two components), $\mathbf{P e}$ is the diffusional Peclet number and measures the relative strengths of advection and diffusion, $M$ is the nondimensional mobility, $\mu$ is the generalized chemical potential, $\phi(c)=F^{\prime}(c)$, and $F(c)$ is the Helmholtz free energy which is nonconvex if $T<T_{c}$, to reflect the coexistence of separate phases and $\epsilon>0$ is a nondimensional measure of non-locality due to the gradient energy (Cahn number) and introduces an internal length scale (interface thickness). See also $[4,32,44]$ for further details and references. The nondimensionalization can be found in [44,40]. Here, for simplicity, we consider a constant mobility ${ }^{1}(M \equiv 1)$ and we take $\mathbf{P e}=1$ and we use the quartic free energy $F(c)$, which is defined by

$$
F(c)=\frac{1}{4} c^{2}(c-1)^{2} .
$$

Thus, the coexisting phases correspond to $c=0$ and 1 . The natural boundary and initial conditions for the $\mathbf{C H}$ equation are

$$
\frac{\partial c}{\partial n}=\frac{\partial \mu}{\partial n}=0, \text { and } \quad \mathbf{u}=\mathbf{0} \quad \text { on } \partial \Omega, \quad c(\mathbf{x}, 0)=c_{0}(\mathbf{x}), \quad \mathbf{u}(\mathbf{x}, t)=\mathbf{0},
$$

Email addresses: jskim@math.umn. edu (Junseok Kim), kkang@mis.mpg.de (Kyungkeun Kang), lowengrb@math . umn . edu (John Lowengrub).

URLs: http: / /www. ima. umn.edu/ junkim/(Junseok Kim), http: //www . math . umn . edu/ lowengrb/ (John Lowengrub).

1 The extension to more general $M=M(c)$ is straightforward and will be considered in a future work [37]. See also Fig. 11 in section 7. 
where $n$ is the normal unit vector pointing out of $\Omega$.

Two important features of the $\mathbf{C H}$ problem in the case of zero Neumann boundary conditions are the conservation of mass $\frac{1}{|\Omega|} \int_{\Omega} c(x, t) d \mathbf{x}$, and the existence of a Lyapunov(Energy) functional $J(c)$

$$
J(c)=\int_{\Omega}\left[F(c)+\frac{\epsilon^{2}}{2}|\nabla c|^{2}\right] d \mathbf{x}
$$

such that

$$
\frac{d}{d t} J(c)=-\int_{\Omega}|\nabla \mu|^{2} d \mathbf{x}
$$

in the absence of flow. The second feature plays a crucial role in the analysis of the CH equation, including the proof of the existence of a solution to the initial boundary value problem [21], and asymptotic long time behavior [54], [58]. The energy functional also readily yields a pointwise estimate of $c$ in the one-dimensional case. In this paper, we develop a finite difference scheme in two dimensions that inherits mass conservation and energy dissipation from the continuous level. It is highly desirable to have a discrete energy functional because this can be used to prove that the numerical solution is uniformly bounded with respect to the time and space step sizes from which it follows that the scheme is stable.

The $\mathbf{C H}$ equation, even without flow, is challenging to solve numerically for two reasons. First, the equation is fourth order in space which makes straightforward difference stencils very large and introduces a severe time step restriction for stability (stiffness), i.e., $\Delta t \sim \Delta x^{4}$ for explicit methods. Second, there is nonlinearity associated with $\phi$, which can also contribute to numerical stiffness. To overcome these difficulties, we split the fourth order equation into a system of second order equations. We then use a fully implicit time discretization. A new nonlinear multigrid method is developed to solve the nonlinear discrete system to obtain the solution at the new time step. We find that convergence of the multigrid method can be achieved with $\Delta t \leq \Delta t_{0}$ where $\Delta t_{0}$ depends only on physical parameters and is independent of the grid size. Since determining the precise value of $\Delta t_{0}$ depends on the application, we find that by taking $\Delta t \sim h$ we obtain convergence in a wide variety of applications. Once this time step restriction is satisfied and the multigrid method converges, our discrete energy readily yields that the overall scheme is stable.

In the presence of flow, the advection term is implemented as a forcing function in the nonlinear multigrid scheme. The functional $J(c)$ now may either increase or decrease in time and satisfies

$$
\frac{d}{d t} J(c)+\int_{\Omega} \mu \nabla \cdot(c \mathbf{u}) d \mathbf{x}=-\int_{\Omega}|\nabla \mu|^{2} d \mathbf{x}
$$


If the interfaces are passive, by which we mean that the concentration field $c$ does not affect the flow field, then $\mathbf{u}$ satisfies the classical Navier-Stokes equations and may be imposed independently of the concentration field $c$. If the interfaces are active, on the other hand, the velocity field $\mathbf{u}$ depends on $c$ through the introduction of extra stresses that mimic the surface tension between the two fluid components. In this case, the system energy is given by

$$
E_{t o t}=\int_{\Omega}|\mathbf{u}|^{2} / 2 d x+\frac{\mathbf{W e}_{s}^{-1}}{\epsilon} J(c),
$$

where the first term is the kinetic energy of the fluid system, the second when scaled this way (note the dependence of the second term upon $\epsilon$ ) is a measure of the surface energy and $\mathbf{W e}_{s}$ is proportional to the Weber number ${ }^{2}$ which measures the relative strengths of the kinetic and surface energies [44]. The velocity satisfies a generalized Navier-Stokes NS system:

$$
\begin{aligned}
\mathbf{u}_{t}+\mathbf{u} \cdot \nabla \mathbf{u} & =-\nabla p-\frac{\mathbf{W e}_{s}^{-1}}{\epsilon} c \nabla \mu+\frac{1}{\mathbf{R e}} \nabla \cdot\left(\eta(c)\left(\nabla \mathbf{u}+\nabla \mathbf{u}^{T}\right)\right) \\
\nabla \cdot \mathbf{u} & =0
\end{aligned}
$$

where the extra stress due to the concentration gradients (i.e. interfaces) is $-\frac{\mathbf{W e}_{s}^{-1}}{\epsilon} c \nabla \mu$, $\mathbf{R e}$ is the Reynolds number and $\eta$ is the nondimensional viscosity which is assumed to depend on the mass concentration $c$. In [44], it is shown using the method of matched asymptotic expansions that this term converges to the classical surface tension force as $\epsilon \rightarrow 0$. This result has been recently made rigorous by C. Liu \& S. Shkoller (preprint) where it is shown that solutions to the NS and $\mathbf{C H}$ equations converge to weak solutions of classical sharp interface models of interfacial flows with surface tension. This NSCH system is known as Model $\mathrm{H}$ in the notation of Hohenberg \& Halperin [32]. We refer the reader to the recent review paper by Anderson, McFadden \& Wheeler [4] and to [44], for example, for further details on the model. The extension of this system to the more realistic case of multiphase fluids whose components have different densities is extensively discussed in [44]. The system energy, assuming no-slip $(\mathbf{u}=0)$ boundary conditions, satisfies

$$
\frac{d}{d t} E_{t o t}=-\frac{\mathbf{W e}_{s}^{-1}}{\epsilon} \int_{\Omega}|\nabla \mu|^{2} d \mathbf{x}-\frac{\mathbf{R e}^{-1}}{2} \int_{\Omega} \eta(c) \mathbf{D}: \mathbf{D} d x
$$

where $\mathbf{D}=\nabla \mathbf{u}+\nabla \mathbf{u}^{T}$ is the scaled deformation tensor.

In this paper, we consider both passive and active interfaces where the generalized NS equations are solved using a second-order accurate finite difference projection method. The resulting discretization of the NSCH system (1-2) and (7)

2 The exact relation is $\mathbf{W e}=\mathbf{W e}_{s} / \int_{0}^{1} \sqrt{2 F(c)} d c$ where We is the physical Weber number [44]. 
(i) preserves the mass (average of $c$ ) on the discrete level;

(ii) gives a simple treatment of boundary conditions;

(iii) has a discrete equivalent of the Lyapunov function $E_{t o t}$;

(iv) has a constraint on the time step which depends only on the physical quantities $\phi, \epsilon, \sigma$ and $\eta$ and not on the spatial discretization;

(v) extends easily to the multi-component case (i.e. two or more concentration fields).

One of the main achievements of this paper is the development of an efficient nonlinear multigrid method to solve the discrete nonlinear scheme for the $\mathbf{C H}$ equation. To our knowledge this is the first work in which a nonlinear multigrid method is used to solve this equation. Following the general strategy outlined in [11], we design a smoother based on treating the $\mathbf{C H}$ equation as a system of two equations for $c$ and $\mu$ as in (1-2). This smoother is of pointwise collective Newton-Gauss-Seidel type [11], in which the nonlinearity is linearized about the current grid point and a Jacobi type update is used for the remaining nonlinear terms. A Gauss-Seidel type update is used for the linear terms and $c$ and $\mu$ are updated simultaneously. We demonstrate the excellent performance of the numerical method by simulating various regimes of the NSCH model including spinodal decomposition.

In the absence of flow, there has been much algorithm development and many simulations of the $\mathbf{C H}$ equation using finite element methods (e.g. [5], [6], [7], [9], [21], [25], [23], [24], [27]), finite difference algorithms (e.g. [26], [28], [29], [53]) and spectral methods (e.g. [41,17]). Most of these finite difference and finite element references use conservative algorithms with discrete energy functionals. The discretization that is closest to ours is given in [21] in the context of finite element methods. In [21], the $\mathbf{C H}$ equation is treated as a system of equations for $c$ and $\mu$ and a Crank-Nicholson type time discretization is used where the free energy derivative $\phi=F^{\prime}(c)$ is approximated so as to yield a scheme with a discrete energy functional for any value of the time step (the scheme is nonlinear at the implicit time level). Here, we choose a different approximation for $\phi$ on the discrete level that yields enhanced stability over the method presented in [21]. This alternative approximation also allows us to extend systematically the discrete system to the case of ternary mixtures; the scheme presented in [21] does not have such a straightforward ternary extension. We will consider ternary fluid flow in another paper [38]. In spite of recent algorithmic developments of numerical approximations to the $\mathbf{C H}$ equation, the solution of the discrete equations has remained problematic due to the nonlinearity of the implicit scheme. By using the nonlinear multigrid method to obtain the numerical solution at the implicit time level, we gain improved numerical stability and efficiency over standard solution techniques based on Newton's method and over algorithms for which the nonlinear term $\phi$ is treated as a forcing function.

In the presence of flow, there has been much recent work on simulating multicomponent fluid flows using Cahn-Hilliard (diffuse interface) models. We again 
refer the reader to the review [4] and to the discussion below for references. To our knowledge, the scheme we present here is the first to have an associated Lyapunov function $E_{t o t}$ on the fully discrete level for any value of the time and space steps. We note that in [33] a semi-discrete NSCH system was presented, where time remained continuous, that did have a Lyapunov function. Recent applications of Cahn-Hilliard fluid modeling include simulations of the two and three dimensional Rayleigh-Taylor instability (e.g. [33,31,43]), the pinchoff of liquid/liquid jets (e.g. [46,42]), thermocapillary flow (e.g. [35,57]), mixing (e.g. [15]), contact angles and wetting phenomena (e.g. [34,49]), gravity and capillary waves (e.g. [3,50,51]), coalescence (e.g. [56]), reactive flows (e.g. [52]), nucleation and spinodal decomposition (e.g. $[18,19,46,35,56,30])$. In particular, in $[30,35]$, the spinodal decomposition occurs in the presence of imposed temperature gradients.

Here, we examine the effect of shear flow and interfacial tension on spinodal decomposition. The solution of the Cahn-Hilliard equation and the resolution of the associated extra stresses in the fluid are typically the bottlenecks of these simulations. Our improved $\mathbf{C H}$ solver together with our energy-preserving discretization for the NSCH system, which has the advantageous side-effect of improving the accuracy of the extra-stress, results in improved stability and accuracy of the multicomponent flow simulations.

The contents of this paper are as follows. In Section 2, we derive the discrete scheme, demonstrate the existence of a discrete energy functional and, in the absence of flow, prove stability and convergence of the algorithm. In Section 3, we present the nonlinear multigrid method for the fully discrete system in the absence of flow. In section 4, we present the approximate projection method used to solve the discrete generalized NS equations. In section 5, we perform a local mode analysis for the nonlinear multigrid scheme to analyze the smoothing factor. Finally in Section 6, we present numerical results for passive and active interfaces. In Appendix A, we present the extension of our scheme to ternary multi-component systems. In Appendix B, the derivation of the smoothing operator for the nonlinear multigrid scheme is presented. In Appendix $\mathrm{C}$, an alternative algorithm for the $\mathbf{C H}$ equation is discussed in which the free energy derivative $\phi$ is not approximated (i.e. the classical Crank-Nicholson discretization).

\section{Numerical analysis}

In this section, we present semi-discrete and fully discrete schemes for the NSCH system. In addition, we prove discrete versions of mass conservation and energy dissipation, which immediately imply the stability of the numerical scheme (assuming that the implicit nonlinear equations can be solved). Finally, the proof of convergence of the numerical solution, in the absence of flow, is also established. 


\subsection{Discretization}

We shall first discretize the $\mathbf{C H}$ equation (1-2) in space. Let $[a, b]$ and $[c, d]$ be partitioned by

$$
\begin{gathered}
a=x_{\frac{1}{2}}<x_{1+\frac{1}{2}}<\cdots<x_{N_{x}-1+\frac{1}{2}}<x_{N_{x}+\frac{1}{2}}=b, \\
c=y_{\frac{1}{2}}<y_{1+\frac{1}{2}}<\cdots<y_{N_{x}-1+\frac{1}{2}}<y_{N_{y}+\frac{1}{2}}=d
\end{gathered}
$$

so that the cells $I_{i j}=\left[x_{i-\frac{1}{2}}, x_{i+\frac{1}{2}}\right] \times\left[y_{j-\frac{1}{2}}, y_{j+\frac{1}{2}}\right], 1 \leq i \leq N_{x}, 1 \leq j \leq N_{y}$ cover $\Omega=[a, b] \times[c, d]$. Let

$$
\Delta x_{i}=x_{i+\frac{1}{2}}-x_{i-\frac{1}{2}}, \quad \Delta y_{j}=y_{j+\frac{1}{2}}-y_{j-\frac{1}{2}} .
$$

For simplicity, we assume that the above partitions are uniform in both directions so that

$$
\Delta x_{i}=\Delta y_{j}=h \quad \text { for } 1 \leq i \leq N_{x}, \quad 1 \leq j \leq N_{y}
$$

where $h=(b-a) / N_{x}=(d-c) / N_{y}$. Therefore

$$
x_{i+\frac{1}{2}}=a+i h, \quad y_{j+\frac{1}{2}}=c+j h
$$

and let $\Omega_{h}=\left\{\left(x_{i}, y_{j}\right): 1 \leq i \leq N_{x}, \quad 1 \leq j \leq N_{y}\right\}$, be the set of cell-centers where

$$
x_{i}=\frac{1}{2}\left(x_{i-\frac{1}{2}}+x_{i+\frac{1}{2}}\right), \quad y_{j}=\frac{1}{2}\left(y_{j-\frac{1}{2}}+y_{j+\frac{1}{2}}\right) .
$$

The set of cell-corners is $\Omega_{h, \frac{1}{2}}=\left\{\left(x_{i+\frac{1}{2}}, y_{j+\frac{1}{2}}\right): 0 \leq i \leq N_{x}, 0 \leq j \leq N_{y}\right\}$.

Since the concentration $c$ and the chemical potential $\mu$ satisfy Neumann boundary conditions, it is natural to define them at cell centers. Let $c_{i j}$ and $\mu_{i j}$ be approximations of $c\left(x_{i}, y_{j}\right)$ and $\mu\left(x_{i}, y_{j}\right)$. We first implement the zero Neumann boundary condition (4) by requiring that

$$
\begin{array}{llll}
D_{x} c_{i-\frac{1}{2}, j}=0 & \text { for } i=0, & D_{x} c_{i+\frac{1}{2}, j}=0 & \text { for } i=N_{x} \\
D_{y} c_{i, j-\frac{1}{2}}=0 & \text { for } j=0, & D_{y} c_{i, j+\frac{1}{2}}=0 & \text { for } j=N_{y}
\end{array}
$$

where the discrete differentiation operators are

$$
D_{x} c_{i+\frac{1}{2}, j}=\frac{1}{h}\left(c_{i+1, j}-c_{i, j}\right), \quad D_{y} c_{i, j+\frac{1}{2}}=\frac{1}{h}\left(c_{i, j+1}-c_{i, j}\right) .
$$

We then define the discrete Laplacian by

$$
\Delta_{d} c_{i j}=\frac{1}{h}\left(D_{x} c_{i+\frac{1}{2}, j}-D_{x} c_{i-\frac{1}{2}, j}\right)+\frac{1}{h}\left(D_{y} c_{i, j+\frac{1}{2}}-D_{y} c_{i, j-\frac{1}{2}}\right)
$$

and the discrete $L^{2}$ inner product by

$$
\left(c_{1}, c_{2}\right)_{h}=h^{2} \sum_{i=1}^{N_{x}} \sum_{j=1}^{N_{y}} c_{1 i j} c_{2 i j}
$$


For a grid function $c$ defined at cell centers, $D_{x} c$ and $D_{y} c$ are defined at cell-edges, and we use the following notation

$$
\nabla_{d}^{e} c_{i j}=\left(D_{x} c_{i+\frac{1}{2}, j}, D_{y} c_{i, j+\frac{1}{2}}\right)
$$

to represent the discrete gradient of $c$ at cell-edges. Correspondingly, the (MAC) divergence at cell-centers, using values from cell-edges, is

$$
\tilde{\nabla}_{d}^{e} \cdot \mathbf{g}_{i j}=\frac{1}{h}\left(g_{i+\frac{1}{2}, j}^{1}-g_{i-\frac{1}{2}, j}^{1}\right)+\frac{1}{h}\left(g_{i, j+\frac{1}{2}}^{2}-g_{i, j-\frac{1}{2}}^{2}\right)
$$

for a grid function $\mathbf{g}=\left(g^{1}, g^{2}\right)$ defined on cell-edges. We can define an inner product for $\nabla_{d}^{e} c$ on the staggered grid by

$$
\left(\nabla_{d}^{e} c_{1}, \nabla_{d}^{e} c_{2}\right)_{e}=h^{2}\left(\sum_{i=0}^{N_{x}} \sum_{j=1}^{N_{y}} D_{x} c_{1+\frac{1}{2}, j} D_{x} c_{2 i+\frac{1}{2}, j}+\sum_{i=1}^{N_{x}} \sum_{j=0}^{N_{y}} D_{y} c_{1, j+\frac{1}{2}} D_{y} c_{2 i, j+\frac{1}{2}}\right)
$$

We also define discrete norms associated with (10) and (11) as

$$
\|c\|^{2}=(c, c)_{h}, \quad|c|_{e, 1}^{2}=\left(\nabla_{d}^{e} c, \nabla_{d}^{e} c\right)_{e} .
$$

The time-continuous, space-discrete system that corresponds to (1-4) in the absence of flow is

$$
\frac{d}{d t} c_{i j}=\Delta_{d} \mu_{i j}, \quad \mu_{i j}=\phi\left(c_{i j}\right)-\epsilon^{2} \Delta_{d} c_{i j},
$$

where $\phi$ is defined in (3) and boundary conditions are implemented using (9). It is easy to see that this discretization is second order accurate in space and that mass is conserved identically. The scheme also has an energy functional given by the discretization of (5). We discretize (12) in time by the Crank-Nicholson type algorithm:

$$
\begin{gathered}
\frac{c_{i j}^{n+1}-c_{i j}^{n}}{\Delta t}=\Delta_{d} \mu_{i j}^{n+\frac{1}{2}} \\
\mu_{i j}^{n+\frac{1}{2}}=\hat{\phi}\left(c_{i j}^{n}, c_{i j}^{n+1}\right)-\frac{\epsilon^{2}}{2} \Delta_{d}\left(c_{i j}^{n}+c_{i j}^{n+1}\right),
\end{gathered}
$$

where

$$
\hat{\phi}\left(c_{1}, c_{2}\right)=\phi\left(c_{2}\right)-\frac{1}{2} \phi^{\prime}\left(c_{2}\right)\left(c_{2}-c_{1}\right)+\frac{1}{3 !} \phi^{\prime \prime}\left(c_{2}\right)\left(c_{2}-c_{1}\right)^{2} .
$$

This is obtained by using the Taylor expansion of $\left(F\left(c_{1}\right)-F\left(c_{2}\right)\right) /\left(c_{1}-c_{2}\right)$ and retaining terms up to the second order derivative. This is a modification of the scheme presented in [21], where $\hat{\phi}\left(c_{1}, c_{2}\right)$ is was taken to be: 


$$
\hat{\phi}\left(c_{1}, c_{2}\right)=\left\{\begin{array}{cl}
\frac{F\left(c_{1}\right)-F\left(c_{2}\right)}{c_{1}-c_{2}}, & \text { if } c_{1} \neq c_{2} \\
\phi\left(c_{1}\right), & \text { if } c_{1}=c_{2} .
\end{array}\right.
$$

And unlike the scheme in [21], our modified scheme can be easily extended to multi-component systems (see Appendix A). In Appendix D, we consider a more traditional Crank-Nicolson discretization in which $\hat{\phi}\left(c_{1}, c_{2}\right)=\left(\phi\left(c_{1}\right)+\phi\left(c_{2}\right)\right) / 2$.

In the presence of flow $\mathbf{u}=(u, v) \neq 0$, we use the center difference operator to define the discrete gradient and divergence operators at cell-centers respectively by

$$
\begin{aligned}
\nabla_{d} c_{i j} & =\frac{1}{2}\left(D_{x} c_{i+\frac{1}{2}, j}+D_{x} c_{i-\frac{1}{2}, j}, D_{y} c_{i, j+\frac{1}{2}}+D_{y} c_{i, j-\frac{1}{2}}\right), \\
\nabla_{d} \cdot \mathbf{u}_{i j} & =\frac{1}{2}\left(D_{x} u_{i+\frac{1}{2}, j}+D_{x} u_{i-\frac{1}{2}, j}\right)+\frac{1}{2}\left(D_{y} v_{i, j+\frac{1}{2}}+D_{y} v_{i, j-\frac{1}{2}}\right) .
\end{aligned}
$$

The non-slip boundary conditions are implemented by introducing a ring of ghostcells surrounding the physical domain such that sum of the velocities (at cellcenters) are equal to zero on the physical boundary, i.e.

$$
\mathbf{u}_{0, j}=-\mathbf{u}_{1, j}, \quad \mathbf{u}_{N_{x}+1, j}=-\mathbf{u}_{N_{x}, j}, \quad \mathbf{u}_{i, 0}=-\mathbf{u}_{i, 1} \text { and } \mathbf{u}_{\mathrm{i}, \mathrm{N}_{\mathrm{y}}+1}=-\mathbf{u}_{\mathrm{i}, \mathrm{N}_{\mathrm{y}}} .
$$

Accordingly, equation (13) becomes by

$$
\frac{c_{i j}^{n+1}-c_{i j}^{n}}{\Delta t}+\nabla_{d} \cdot\left(\mathbf{u}_{i j}^{n+\frac{1}{2}} c_{i j}^{n+\frac{1}{2}}\right)=\Delta_{d} \mu_{i j}^{n+\frac{1}{2}}
$$

where $\mathbf{u}^{n+\frac{1}{2}}=\left(\mathbf{u}^{n+1}+\mathbf{u}^{n}\right) / 2$, and $c^{n+\frac{1}{2}}$ is defined analogously. The chemical potential $\mu_{i j}^{n+\frac{1}{2}}$ is still obtained from (14).

For active interfaces, the velocity $\mathbf{u}$ satisfies the generalized Navier-Stokes equations (7). Here, we will use the rotation form (i.e. $\mathbf{u} \cdot \nabla \mathbf{u}=\omega \times \mathbf{u}+\frac{1}{2} \nabla|\mathbf{u}|^{2}$ ) of the equations, together with the Bernoulli pressure $P=p+\frac{1}{2}|\mathbf{u}|^{2}$. To solve this system, we will use a projection method. Following [1], the velocity components are defined at cell centers where $\mathbf{u}_{i j} \approx \mathbf{u}\left(x_{i}, y_{j}\right)$ and the pressure is defined at cellcorners $\Omega_{h, \frac{1}{2}}$ where $P_{i+\frac{1}{2}, j+\frac{1}{2}} \approx P\left(x_{i+\frac{1}{2}}, y_{j+\frac{1}{2}}\right)$. In the corresponding discrete system, we additionally define gradient and divergence operators taking values from cell-centers to cell-corners and vice-versa. These operators are:

$$
\begin{gathered}
\nabla_{d}^{c} \cdot \mathbf{u}_{i+\frac{1}{2}, j+\frac{1}{2}}=\frac{1}{2}\left(D_{x} u_{i+\frac{1}{2}, j}+D_{x} u_{i+\frac{1}{2}, j+1}\right)+\frac{1}{2}\left(D_{y} v_{i, j+\frac{1}{2}}+D_{y} v_{i+1, j+\frac{1}{2}}\right), \\
\tilde{\nabla}_{d}^{c} P_{i, j}=\frac{1}{2 h}\left(P_{i+\frac{1}{2}, j+\frac{1}{2}}+P_{i+\frac{1}{2}, j-\frac{1}{2}}-P_{i-\frac{1}{2}, j+\frac{1}{2}}-P_{i-\frac{1}{2}, j-\frac{1}{2}},\right. \\
\left.P_{i+\frac{1}{2}, j+\frac{1}{2}}-P_{i+\frac{1}{2}, j-\frac{1}{2}}+P_{i-\frac{1}{2}, j+\frac{1}{2}}-P_{i-\frac{1}{2}, j-\frac{1}{2}}\right) .
\end{gathered}
$$


We also introduce two Laplace operators that operate on cell-corners:

$$
\Delta_{d}^{c} \psi_{i+\frac{1}{2}, j+\frac{1}{2}}=\frac{\psi_{i-\frac{1}{2}, j+\frac{1}{2}}+\psi_{i+\frac{1}{2}, j+\frac{3}{2}}-4 \psi_{i+\frac{1}{2}, j+\frac{1}{2}}+\psi_{i+\frac{1}{2}, j-\frac{1}{2}}+\psi_{i+\frac{3}{2}, j+\frac{1}{2}}}{h^{2}}
$$

and

$$
\nabla_{d}^{c} \cdot \tilde{\nabla}_{d}^{c} \psi_{i+\frac{1}{2}, j+\frac{1}{2}}=\frac{\psi_{i-\frac{1}{2}, j-\frac{1}{2}}+\psi_{i+\frac{3}{2}, j+\frac{1}{2}}-4 \psi_{i+\frac{1}{2}, j+\frac{1}{2}}+\psi_{i-\frac{1}{2}, j-\frac{3}{2}}+\psi_{i+\frac{3}{2}, j+\frac{3}{2}}}{2 h^{2}} .
$$

To discretize the flow equations, we then use an approximate projection method (e.g. see $[1,2])$. In this scheme, the incompressibility condition can be posed as

$$
\nabla_{d}^{c} \cdot\left(\frac{\mathbf{u}^{n+1}-\mathbf{u}^{n}}{\Delta t}\right)=\Delta t\left(\Delta_{d}^{c}-\nabla_{d}^{c} \cdot \tilde{\nabla}_{d}^{c}\right) \mathcal{L}^{-1}\left(\frac{p^{n+\frac{1}{2}}-p^{n-\frac{1}{2}}}{\Delta t}\right)
$$

where $\mathcal{L}=I-\frac{\Delta t \eta}{2 \operatorname{Re}} \Delta_{d}^{c}$ and $I$ is the identity operator. This form of the approximate projection, to our knowledge, has not been given in other papers utilizing the projection method. See section 4 for a derivation. Note that the difference $\Delta_{d}^{c}-\nabla_{d}^{c} \cdot \tilde{\nabla}_{d}^{c}$ applied to a smooth function converges to zero with second order accuracy. If $\Delta_{d}^{c}$ was taken to be $\nabla_{d}^{c} \cdot \tilde{\nabla}_{d}^{c}$, then the velocity is divergence-free on the discrete level. However, the corresponding exact projection method is ill-conditioned since the stencil for the pressure solve is algebraically decoupled (e.g. see [45]).

Finally, the discretized momentum equation is

$$
\begin{aligned}
& \frac{\mathbf{u}_{i j}^{n+1}-\mathbf{u}_{i j}^{n}}{\Delta t}=-\omega_{i j}^{n+\frac{1}{2}} \times \mathbf{u}_{i j}^{n+\frac{1}{2}}-\tilde{\nabla}_{d}^{c} P_{i j}^{n+\frac{1}{2}}+\delta \mathbf{u}_{i j}^{n+\frac{1}{2}} \\
&-\frac{\mathbf{W e}_{s}^{-1}}{\epsilon} c_{i j}^{n+\frac{1}{2}} \nabla_{d} \mu_{i j}^{n+\frac{1}{2}}+\frac{1}{\mathbf{R e}} \nabla_{d} \cdot\left(\eta\left(c_{i j}^{n+\frac{1}{2}}\right) \mathbf{D}_{i j}^{n+\frac{1}{2}}\right) \\
&+\frac{\Delta t^{2}}{2 \mathbf{R e}} \nabla_{d} \cdot\left(\eta\left(c^{n+\frac{1}{2}}\right)\left(\nabla_{d} \tilde{\nabla}_{d}^{c}+\nabla_{d}^{T} \tilde{\nabla}_{d}^{c}-\Delta_{d}^{c} I\right) \mathcal{L}^{-1}\left(\frac{P^{n+\frac{1}{2}}-P^{n-\frac{1}{2}}}{\Delta t}\right)\right)
\end{aligned}
$$

where the vorticity is $\omega_{i j}^{n+\frac{1}{2}}=\nabla_{d} \times \mathbf{u}_{i j}^{n+\frac{1}{2}}$, and the (scaled) rate of deformation tensor is $\mathbf{D}_{i j}^{n+\frac{1}{2}}=\nabla_{d} \mathbf{u}_{i j}^{n+\frac{1}{2}}+\left(\nabla_{d} \mathbf{u}_{i j}^{n+\frac{1}{2}}\right)^{T}$. The last term in Eq. (19) arises in the projection method by replacing an intermediate velocity that consists of the projected velocity at the $n+1$ step and a pressure update. Again, this form of the projection method has not been presented in other papers utilizing the projection method. Again, see section 4 for a derivation. If the viscosity $\eta$ is constant, the latter two viscous terms in Eq. (19) are replaced by the single term $\mathbf{R e}^{-1} \Delta_{d} \mathbf{u}^{n+\frac{1}{2}}$. We include the more general case here for completeness although in this paper we focus on the case in which $\eta$ constant. The case with non-constant $\eta$ will be considered in another paper [37]. 
If $\eta$ is constant and one uses the replacement described above, then $\delta$ in Eq. (19) is given by

$$
\delta=\left(\mathbf{u}^{n+\frac{1}{2}}, \tilde{\nabla}_{d}^{c} P^{n+\frac{1}{2}}\right)_{h} /\left\|\mathbf{u}^{n+\frac{1}{2}}\right\|^{2}
$$

in order to remove the components of $\tilde{\nabla}_{d}^{c} P_{i j}^{n+\frac{1}{2}}$ that are not orthogonal to $\mathbf{u}_{i j}^{n+\frac{1}{2}}$ in the discrete $L^{2}$ space. Note that on the continuous level, $\delta=0$. Indeed, as we demonstrate in section $6, \delta$ converges to zero with second order accuracy. On the discrete level this term ensures, as we will demonstrate in the next section, that the discrete system (17)-(19) in fact has an energy functional given by the discretization of (6). For non-constant $\eta$, the projection factor should be taken to be [37]:

$$
\begin{aligned}
\delta= & \left(\mathbf{u}^{n+\frac{1}{2}}, \tilde{\nabla}_{d}^{c} P^{n+\frac{1}{2}}\right)_{h} /\left\|\mathbf{u}^{n+\frac{1}{2}}\right\|^{2} \\
& -\frac{\left(\mathbf{u}^{n+\frac{1}{2}}, \nabla_{d} \cdot \eta\left(c^{n+\frac{1}{2}}\right)\left(\nabla_{d} \tilde{\nabla}_{d}^{c}+\nabla_{d}^{T} \tilde{\nabla}_{d}^{c}-\Delta_{d}^{c} I\right) \mathcal{L}^{-1}\left(\frac{P^{n+\frac{1}{2}}-P^{n-\frac{1}{2}}}{\Delta t}\right)\right)_{h}}{2 \Delta t^{-2} \mathbf{R e}\left\|\mathbf{u}^{n+\frac{1}{2}}\right\|^{2}} .
\end{aligned}
$$

The numerical implementation of Eqs. (13)-(14) and (18)- (20) is discussed in sections 3 and 4 where it is shown how the implicit solutions at time $t_{n+1}$ are obtained using multigrid methods.

\subsection{Analysis of Scheme}

We next analyze the numerical schemes. At this stage, we assume there are no restrictions to solving the implicit systems of equations. Later, we examine the effectiveness of the multigrid algorithms used to solve the implicit equations. Before we proceed, we first state without proof the following lemma which is a easy consequence of discrete summation by parts.

Lemma 1 Let $c_{1}$ and $c_{2}$ be defined on $\Omega_{h}$ satisfying (9). Let $\mathbf{u}$ be defined on $\Omega_{h}$ and satisfy (16). Finally, let $P$ be defined on $\Omega_{h, \frac{1}{2}}$. Then,

$$
\begin{aligned}
& \left(c_{1}, \Delta_{d} c_{2}\right)_{h}=\left(\Delta_{d} c_{1}, c_{2}\right)_{h}=-\left(\nabla_{d}^{e} c_{1}, \nabla_{d}^{e} c_{2}\right)_{e} \\
& \left(\mathbf{u}, \nabla_{d} c_{1}\right)_{h}=-\left(\nabla_{d} \cdot \mathbf{u}, c_{1}\right)_{h}, \text { and } \\
& \left(\mathbf{u}, \tilde{\nabla}_{d}^{c} P\right)_{h}=-\left(\nabla_{d}^{c} \cdot \mathbf{u}, P\right)_{h, \frac{1}{2}}
\end{aligned}
$$

where the cell-corner inner product is given by the trapezoidal rule approximation of the continuous inner product:

$$
\left(\nabla_{d}^{c} \cdot \mathbf{u}, P\right)_{h, \frac{1}{2}}=\frac{h^{2}}{2} \sum_{i=0}^{N_{x}} \sum_{j=0}^{N_{y}} \nabla_{d}^{c} \cdot \mathbf{u}_{i+\frac{1}{2}, j+\frac{1}{2}} P_{i+\frac{1}{2}, j+\frac{1}{2}}+\frac{h^{2}}{2} \sum_{i=1}^{N_{x}-1} \sum_{j=1}^{N_{y}-1} \nabla_{d}^{c} \cdot \mathbf{u}_{i+\frac{1}{2}, j+\frac{1}{2}} P_{i+\frac{1}{2}, j+\frac{1}{2}}
$$


The following lemma establishes the mass conservation and the existence of a discrete energy functional in the absence of flow.

Lemma 2 If $\left\{c^{n}, \mu^{n-\frac{1}{2}}\right\}$ is the solution of (13-14) and if we define the discrete energy functional by

$$
\mathcal{F}_{h}(c)=(F(c), 1)_{h}+\frac{\epsilon^{2}}{2}|c|_{e, 1}^{2},
$$

where $F$ is defined in (3), then

$$
\left(c^{n+1}, 1\right)_{h}=\left(c^{n}, 1\right)_{h},
$$

and

$$
\mathcal{F}_{h}\left(c^{n+1}\right)-\mathcal{F}_{h}\left(c^{n}\right)=-\Delta t\left|\mu^{n+\frac{1}{2}}\right|_{e, 1}^{2}-\frac{1}{4}\left(\left(c^{n+1}-c^{n}\right)^{4}, 1\right)_{h}
$$

PROOF. The first assertion is due to the combination of (13) and the discrete version of integration by parts in lemma 1 . Indeed,

$$
\left(c^{n+1}, 1\right)_{h}=\left(c^{n}, 1\right)_{h}+\Delta t\left(\Delta_{d} \mu^{n+\frac{1}{2}}, 1\right)_{h}=\left(c^{n}, 1\right)_{h}-\Delta t\left(\nabla_{d} \mu^{n+\frac{1}{2}}, \nabla_{d} 1\right)_{e}=\left(c^{n}, 1\right)_{h}
$$

It remains to prove the second assertion. Multiplying $\mu^{n+\frac{1}{2}}$ and $c^{n+1}-c^{n}$ to (13) and (14), respectively and summing by parts, we obtain the following two identities

$$
\begin{gathered}
\left(c^{n+1}-c^{n}, \mu^{n+\frac{1}{2}}\right)_{h}+\Delta t\left|\mu^{n+\frac{1}{2}}\right|_{e, 1}^{2}=0 \\
\left(c^{n+1}-c^{n}, \mu^{n+\frac{1}{2}}\right)_{h}-\frac{\epsilon^{2}}{2}\left|c^{n+1}\right|_{e, 1}^{2}+\frac{\epsilon^{2}}{2}\left|c^{n}\right|_{e, 1}^{2}=\left(\hat{\phi}\left(c^{n}, c^{n+1}\right), c^{n+1}-c^{n}\right)_{h} .
\end{gathered}
$$

Using the identities above, we obtain

$$
\begin{aligned}
\mathcal{F}_{h}\left(c^{n+1}\right)-\mathcal{F}_{h}\left(c^{n}\right)= & \frac{\epsilon^{2}}{2}\left|c^{n+1}\right|_{e, 1}^{2}-\frac{\epsilon^{2}}{2}\left|c^{n}\right|_{e, 1}^{2}+\left(F\left(c^{n+1}\right)-F\left(c^{n}\right), 1\right)_{h} \\
= & -\Delta t\left|\mu^{n+\frac{1}{2}}\right|_{e, 1}^{2}-\left(\hat{\phi}\left(c^{n}, c^{n+1}\right), c^{n+1}-c^{n}\right)_{h} \\
& +\left(F\left(c^{n+1}\right)-F\left(c^{n}\right), 1\right)_{h} .
\end{aligned}
$$

From the Taylor expansion, we have

$$
\begin{aligned}
F\left(c^{n+1}\right)-F\left(c^{n}\right)= & \phi\left(c^{n+1}\right)\left(c^{n+1}-c^{n}\right)-\frac{1}{2} \phi^{\prime}\left(c^{n+1}\right)\left(c^{n+1}-c^{n}\right)^{2} \\
& +\frac{1}{3 !} \phi^{\prime \prime}\left(c^{n+1}\right)\left(c^{n+1}-c^{n}\right)^{3}-\frac{1}{4 !} \phi^{\prime \prime \prime}\left(c^{n+1}\right)\left(c^{n+1}-c^{n}\right)^{4} .
\end{aligned}
$$


Since $\phi^{\prime \prime \prime}\left(c^{n+1}\right)=6$, we obtain

$$
\begin{aligned}
& \mathcal{F}_{h}\left(c^{n+1}\right)-\mathcal{F}_{h}\left(c^{n}\right)+\Delta t\left|\mu^{n+\frac{1}{2}}\right|_{e, 1}^{2} \\
= & -\left(\hat{\phi}\left(c^{n}, c^{n+1}\right), c^{n+1}-c^{n}\right)_{h}+\left(F\left(c^{n+1}\right)-F\left(c^{n}\right), 1\right)_{h} \\
= & -\frac{6}{4 !}\left(\left(c^{n+1}-c^{n}\right)^{4}, 1\right)_{h}=-\frac{1}{4}\left(\left(c^{n+1}-c^{n}\right)^{4}, 1\right)_{h} .
\end{aligned}
$$

This completes the proof.

Remark 3 From lemma 2, it follows that the numerical solution $\left\|c^{n}\right\|$ is bounded (assuming that it is possible to solve the nonlinear scheme at the implicit time level). This yields stability (in discrete $l_{2}$ ) of the numerical scheme. The solution of the equations at the implicit time level is discussed in sections 3 and 5.1 where, respectively, the nonlinear multigrid is presented and the weak time step restrictions $\Delta t \leq \Delta t_{0}(\epsilon, \phi)$ or $\Delta t \sim h$ are shown to be sufficient to obtain convergence of the nonlinear multigrid method.

Remark 4 The presence of the second term on the right hand side of Eq. (26) suggests that our method is more stable than that of [21] where this term is absent.

Remark 5 Lemma 2 still holds for regular solution model free energies of the form [8]:

$$
F(c):=\theta[c \ln (c)+(1-c) \ln (1-c)]-2 \theta_{c} c(1-c),
$$

( $\theta$ and $\theta_{c}$ are the absolute and the critical temperatures, respectively) provided that they are regularized by fourth order polynomials near the singular points, i.e.,

$$
F_{\delta}(c)=\left\{\begin{array}{l}
p_{l}(c) \text { if } c \leq \delta, \\
F(c) \text { if } \delta<c<1-\delta, \\
p_{r}(c) \text { if } c \geq 1-\delta,
\end{array}\right.
$$

where $p_{l}(c)$ and $p_{r}(c)$ are fourth order polynomials which match values with $F(c)$ up to fourth order derivatives at $c=\delta$ and $c=1-\delta$, respectively and $\delta$ is a small positive parameter.

Next, we demonstrate the existence of an energy functional in the presence of flow. For simplicity, we state and prove the lemma for the case in which the viscosity $\eta$ is constant.

Lemma 6 Let $\left\{c^{n}, \mu^{n-\frac{1}{2}}, \mathbf{u}^{n}\right\}$ be the solutions of (14) and (17), and (18)-(19) with $\eta$ constant and let the discrete total energy functional be

$$
\mathcal{E}_{h}(c, \mathbf{u})=\frac{1}{2}(\mathbf{u}, \mathbf{u})_{h}+\frac{\mathbf{W e}_{s}^{-1}}{\epsilon}\left((F(c), 1)_{h}+\frac{\epsilon^{2}}{2}|c|_{1}^{2}\right),
$$


then

$$
\begin{aligned}
\frac{\mathcal{E}_{h}\left(c^{n+1}, \mathbf{u}^{n+1}\right)-\mathcal{E}_{h}\left(c^{n}, \mathbf{u}^{n}\right)}{\Delta t}= & -\frac{1}{\mathbf{R e}}\left(\left|u^{n+\frac{1}{2}}\right|_{e, 1}^{2}+\left|v^{n+\frac{1}{2}}\right|_{e, 1}^{2}\right) \\
& -\frac{\mathbf{W e}_{s}^{-1}}{\epsilon}\left|\mu^{n+\frac{1}{2}}\right|_{e, 1}^{2}-\frac{\mathbf{W e}_{s}^{-1}}{4 \epsilon \Delta t}\left(\left(c^{n+1}-c^{n}\right)^{4}, 1\right)_{h}
\end{aligned}
$$

PROOF. Multiply Eq. (19) by $\mathbf{u}^{n+\frac{1}{2}}$ and sum over cell-centers to get

$$
\begin{gathered}
\frac{\left(\mathbf{u}^{n+1}, \mathbf{u}^{n+1}\right)_{h}-\left(\mathbf{u}^{n}, \mathbf{u}^{n}\right)_{h}}{2 \Delta t}=-\left(\mathbf{u}^{n+\frac{1}{2}}, \tilde{\nabla}_{d}^{c} P^{n+\frac{1}{2}}\right)_{h}+\delta\left(\mathbf{u}^{n+\frac{1}{2}}, \mathbf{u}^{n+\frac{1}{2}}\right)_{h} \\
-\frac{\mathbf{W e}_{s}^{-1}}{\epsilon}\left(\mathbf{u}^{n+\frac{1}{2}} c^{n+\frac{1}{2}}, \nabla_{d} \mu^{n+\frac{1}{2}}\right)_{h}+\frac{1}{\mathbf{R e}}\left(\mathbf{u}^{n+\frac{1}{2}}, \Delta_{d} \mathbf{u}^{n+\frac{1}{2}}\right)_{h} \\
\left.=\frac{\mathbf{W e}_{s}^{-1}}{\epsilon}\left(\nabla_{d} \cdot\left(\mathbf{u}^{n+\frac{1}{2}} c^{n+\frac{1}{2}}\right), \mu^{n+\frac{1}{2}}\right)_{h}-\left.\frac{1}{\mathbf{R e}}\left(\left|u^{n+\frac{1}{2}}\right|_{e, 1}^{2}+\mid v^{n+\frac{1}{2}}\right)\right|_{e, 1} ^{2}\right)
\end{gathered}
$$

where we have used the value of $\delta$ from Eq. (20) and we have summed by parts using lemma 1 . Next, multiply Eq. (13) by $\mu^{n+\frac{1}{2}}$ and sum to get

$$
\begin{aligned}
\left(c^{n+1}-c^{n}, \mu^{n+\frac{1}{2}}\right)_{h} & =-\Delta t\left(\nabla_{d} \cdot\left(c^{n+\frac{1}{2}} \mathbf{u}^{n+\frac{1}{2}}\right), \mu^{n+\frac{1}{2}}\right)_{h}+\Delta t\left(\Delta_{d} \mu^{n+\frac{1}{2}}, \mu^{n+\frac{1}{2}}\right)_{h} \\
& =-\Delta t\left(\nabla_{d} \cdot\left(c^{n+\frac{1}{2}} \mathbf{u}^{n+\frac{1}{2}}\right), \mu^{n+\frac{1}{2}}\right)_{h}-\Delta t\left|\mu^{n+\frac{1}{2}}\right|_{e, 1}^{2} .
\end{aligned}
$$

Combining Eqs. (29) and (30) and using the argument in the proof of lemma 2, we obtain

$$
\begin{aligned}
\mathcal{E}_{h}\left(c^{n+1}, \mathbf{u}^{n+1}\right)-\mathcal{E}_{h}\left(c^{n}, \mathbf{u}^{n}\right)=-\frac{\Delta t}{\mathbf{R e}}\left(\left|u^{n+\frac{1}{2}}\right|_{e, 1}^{2}+\left|v^{n+\frac{1}{2}}\right|_{e, 1}^{2}\right) \\
-\frac{\mathbf{W e}_{s}^{-1} \Delta t}{\epsilon}\left|\mu^{n+\frac{1}{2}}\right|_{e, 1}^{2}-\frac{\mathbf{W e}_{s}^{-1}}{4 \epsilon}\left(\left(c^{n+1}-c^{n}\right)^{4}, 1\right)_{h},
\end{aligned}
$$

where we have used that the terms that couple flow with concentration in the equations cancel one another exactly. This completes the proof of the lemma.

Remark 7 From lemma 6, it follows that both $\left\|c^{n}\right\|$ and $\left\|\mathbf{u}^{n}\right\|$ are bounded uniformly in $n$. This yields stability (in discrete $l_{2}$ ) of the numerical scheme in the presence of flow where again we have assumed that it is possible to solve the equations at the implicit time level.

Remark 8 Using the more general discretization (19) and the choice of $\delta$ given in 21 , the lemma also holds if the viscosity $\eta$ is not constant. 
Next, we demonstrate the convergence of the scheme at a fixed time in the absence of flow. Let $\mathbf{u}=\mathbf{0}$ and let $C^{n}$ and $c^{n}$ be the continuous and discrete solutions of Eqs. (1)-(2) and (13)-(14) at time $t=t_{n}$, respectively and let $e^{n}=C^{n}-c^{n}$ be the error. Then we have the following error estimate.

Theorem 9 Suppose $C$ is smooth and $\mathbf{u}=\mathbf{0}$. Then, for any $T>0$, there exists a constant $K, \Delta t_{0}$, and $h_{0}$ depending on $T, \phi, \hat{\phi}, \epsilon$, and smoothness of $C$ such that the following error estimate holds:

$$
\left\|e^{n}\right\| \leq K\left(h^{2}+\Delta t^{2}\right)
$$

for $n \Delta t \leq T$ if $h \leq h_{0}$ and $\Delta t \leq \Delta t_{0}$.

PROOF. Using the numerical scheme, we obtain

$$
\begin{aligned}
\partial_{t} e^{m}+\epsilon^{2} & \Delta_{d}^{2} e^{m+\frac{1}{2}}=\partial_{t} C^{m}+\epsilon^{2} \Delta_{d}^{2} C^{m+\frac{1}{2}}-\Delta_{d} \hat{\phi}\left(c^{m}, c^{m+1}\right) \\
& =C_{t}\left(t_{m+\frac{1}{2}}\right)+\epsilon^{2} \Delta^{2} C\left(t_{m+\frac{1}{2}}\right)-\Delta_{d} \hat{\phi}\left(c^{m}, c^{m+1}\right)+O\left(h^{2}+\Delta t^{2}\right) \\
& =\Delta_{d} \phi\left(C^{m+\frac{1}{2}}\right)-\Delta_{d} \hat{\phi}\left(c^{m}, c^{m+1}\right)+O\left(h^{2}+\Delta t^{2}\right)
\end{aligned}
$$

where by $\partial_{t}$, we denote the temporal difference quotient. From now on, the capital letter $K$ will be used to denote the generic constant, the value of which may change from line to line. Adding and subtracting $\Delta_{d} \phi\left(c^{m+\frac{1}{2}}\right)$ in (31), we obtain

$$
\partial_{t} e^{m}+\epsilon^{2} \Delta_{d}^{2} e^{m+\frac{1}{2}}=\Delta_{d}(A+B)+O\left(h^{2}+\Delta t^{2}\right)
$$

where $A$ and $B$ are defined as follows:

$$
A \equiv \phi\left(C^{m+\frac{1}{2}}\right)-\phi\left(c^{m+\frac{1}{2}}\right), \quad B \equiv \phi\left(c^{m+\frac{1}{2}}\right)-\hat{\phi}\left(c^{m}, c^{m+1}\right) .
$$

Multiplying $e^{m+\frac{1}{2}}$ and using summation by parts, we have

$$
\begin{aligned}
& \frac{1}{2} \partial_{t}\left\|e^{m}\right\|^{2}+\epsilon^{2}\left\|\Delta_{d} e^{m+\frac{1}{2}}\right\|^{2} \leq\left(A, \Delta_{d} e^{m+\frac{1}{2}}\right)_{h}+\left(B, \Delta_{d} e^{m+\frac{1}{2}}\right)_{h} \\
& +K\left(h^{4}+\Delta t^{4}\right)+\left\|e^{m+\frac{1}{2}}\right\|^{2} .
\end{aligned}
$$

We first consider the first term of the right side of (32). Since $\left\|u^{n}\right\|$ and $\left\|c^{n}\right\|$ are bounded, we obtain

$$
\left(A, \Delta_{d} e^{m+\frac{1}{2}}\right)_{h} \leq K\left(\left|e^{m+\frac{1}{2}}\right|,\left|\Delta_{d} e^{m+\frac{1}{2}}\right|\right)_{h} \leq K|| e^{m+\frac{1}{2}}\left\|^{2}+\frac{\epsilon^{2}}{4}\right\| \Delta_{d} e^{m+\frac{1}{2}} \|^{2}
$$


It remains to estimate the second term. A simple computation shows that $B$ is factored as follows:

$$
B=\frac{1}{8}\left(c^{m+1}-c^{m}\right)^{2}\left(3 c^{m+1}-c^{m}-1\right) .
$$

With the aid of the factorization and Young's inequality we obtain

$$
\left(B, \Delta_{d} e^{m+\frac{1}{2}}\right)_{h} \leq K\|B\|^{2}+\frac{\epsilon^{2}}{4}\left\|\Delta_{d} e^{m+\frac{1}{2}}\right\|^{2} \leq K\left\|\left(c^{m+1}-c^{m}\right)^{2}\right\|^{2}+\frac{\epsilon^{2}}{4}\left\|\Delta_{d} e^{m+\frac{1}{2}}\right\|^{2},
$$

where we used the fact that numerical solution is bounded. The next step is to estimate $\left\|\left(c^{m+1}-c^{m}\right)^{2}\right\|^{2}$. Adding and subtracting the analytic solution, we have

$$
\begin{aligned}
\left\|\left(c^{m+1}-c^{m}\right)^{2}\right\|^{2} & \leq 2\left(\left\|\left(c^{m+1}-c^{m}\right)^{2}-\left(C^{m+1}-C^{m}\right)^{2}\right\|^{2}+\left\|\left(C^{m+1}-C^{m}\right)^{2}\right\|^{2}\right) \\
& \leq K\left(\left\|e^{m+1}-e^{m}\right\|^{2}+\left\|\left(C^{m+1}-C^{m}\right)^{2}\right\|^{2}\right)
\end{aligned}
$$

where again the boundedness of the numerical and analytical solutions is used. Since analytic solution $u$ is smooth, the second term is estimated as follows:

$$
\left\|\left(C^{m+1}-C^{m}\right)^{2}\right\|^{2} \leq K \Delta t^{4}\left\|C_{t}\right\|_{\infty}^{4}
$$

Putting everything together, we get

$$
\left(B, \Delta_{d} e^{m+\frac{1}{2}}\right)_{h} \leq K \Delta t^{4}+K\left\|e^{m+1}-e^{m}\right\|^{2}+\frac{\epsilon^{2}}{4}\left\|\Delta_{d} e^{m+\frac{1}{2}}\right\|^{2}
$$

Subtracting $\frac{\epsilon^{2}}{2}\left\|\Delta_{d} e^{m+\frac{1}{2}}\right\|_{2}^{2}$ and multiplying 2 to both sides in (32), we obtain

$$
\partial_{t}\left\|e^{m}\right\|^{2}+\left.\epsilon^{2}\left\|\Delta_{d} e^{m+\frac{1}{2}}\right\|\right|^{2} \leq K\left(h^{4}+\Delta t^{4}\right)+K\left\|e^{m+\frac{1}{2}}\right\|^{2}+K\left\|e^{m+1}-e^{m}\right\|^{2} .
$$

Dropping $\epsilon^{2}\left\|\Delta_{d} e^{m+\frac{1}{2}}\right\|^{2}$ in (33) and summing in time, we have

$$
\begin{aligned}
\left\|e^{n}\right\|^{2} \Delta t^{-1} & \leq \sum_{m=0}^{n-1}\left[K\left(h^{4}+\Delta t^{4}\right)+K\left\|e^{m+\frac{1}{2}}\right\|^{2}+K\left\|e^{m+1}-e^{m}\right\|^{2}\right] \\
& \leq \sum_{m=0}^{n-1}\left[K\left(h^{4}+\Delta t^{4}\right)+K\left\|e^{m+1}\right\|^{2}+K\left\|e^{m}\right\|^{2}\right] \\
& =K n\left(h^{4}+\Delta t^{4}\right)+K \sum_{m=0}^{n-1}\left\|e^{m}\right\|^{2}+K\left\|e^{n}\right\| .
\end{aligned}
$$


Multiplying $\Delta t$ on both sides, we obtain

$$
\begin{aligned}
(1-K \Delta t)\left\|e^{n}\right\|^{2} & \leq K(\Delta t n)\left(h^{4}+\Delta t^{4}\right)+K \Delta t \sum_{m=0}^{n-1}\left\|e^{m}\right\|^{2} \\
& \leq K T\left(h^{4}+\Delta t^{4}\right)+K \Delta t \sum_{m=0}^{n-1}\left\|e^{m}\right\|^{2}
\end{aligned}
$$

where we used the fact that $n \Delta t \leq T$. Since $\Delta t$ can be chosen such that $1-K \Delta t>$ 0 , the discrete version of Gronwall's inequality enables us to obtain $\left\|e^{n}\right\| \leq K\left(h^{2}+\right.$ $\left.\Delta t^{2}\right)$. This completes the proof.

Remark 10 The proof of convergence of the coupled NSCH scheme (14) and (17), and (18)-(19) in the presence of flow is currently under study.

\section{Solution of the system in absence of flow}

In this section, we develop a nonlinear Full Approximation Storage (FAS) multigrid method to solve the nonlinear discrete $\mathbf{C H}$ system (13)-(14) at the implicit time level in the absence of flow. The fundamental idea of nonlinear multigrid is analogous to the linear case. First, the errors to the solution have to be smoothed so that they can be approximated on a coarser grid. An analogue of the linear defect equation is transformed to the coarse grid. The coarse grid corrections are interpolated back to the fine grid, where the errors are again smoothed. However, because the system is nonlinear we do not work with the errors, but rather with full approximations to the discrete solution on the coarse grid. The nonlinearity is treated using one step of Newton's iteration and a pointwise Gauss-Seidel relaxation scheme is used as the smoother in the multigrid method. This corresponds to a local rather than global linearization of the nonlinear scheme and as such is more efficient than standard Newton-Gauss-Seidel global linearization schemes. See the reference text [55] for additional details and background.

Let us rewrite equations (13), (14) as follows.

$$
\mathbf{N S O}\left(c^{n}, c^{n+1}, \mu^{n+\frac{1}{2}}\right)=\left(f^{n}, g^{n}\right)
$$

where

$$
\mathbf{N S O}\left(c^{n}, c^{n+1}, \mu^{n+\frac{1}{2}}\right)=\left(\frac{c_{i j}^{n+1}}{\Delta t}-\Delta_{d} \mu_{i j}^{n+\frac{1}{2}}, \mu_{i j}^{n+\frac{1}{2}}-\hat{\phi}\left(c_{i j}^{n}, c_{i j}^{n+1}\right)+\frac{\epsilon^{2}}{2} \Delta_{d} c_{i j}^{n+1}\right)
$$

and the source term is 


$$
\left(f^{n}, g^{n}\right)=\left(\frac{c_{i j}^{n}}{\Delta t},-\frac{\epsilon^{2}}{2} \Delta_{d} c_{i j}^{n}\right)
$$

In the following description of one FAS cycle, we assume a sequence of grids $\Omega_{k}$ ( $\Omega_{k-1}$ is coarser than $\Omega_{k}$ by factor 2). Given the number $\nu$ of pre- and post- smoothing relaxation sweeps, an iteraton step for the nonlinear multigrid method using the V-cycle is formally written as follows:

\section{FAS multigrid cycle}

$$
\left\{c_{k}^{m+1}, \mu_{k}^{m+\frac{1}{2}}\right\}=F A S \operatorname{cycle}\left(k, c_{k}^{n}, c_{k}^{m}, \mu_{k}^{m-\frac{1}{2}}, \mathbf{N S O}_{k}, f_{k}^{n}, g_{k}^{n}, \nu\right) .
$$

That is, $\left\{c_{k}^{m}, \mu_{k}^{m-\frac{1}{2}}\right\}$ and $\left\{c_{k}^{m+1}, \mu_{k}^{m+\frac{1}{2}}\right\}$ are the approximations of $c_{k}\left(x_{i}, y_{j}\right)$ and $\mu_{k}\left(x_{i}, y_{j}\right)$ before and after a FAScycle. Now, we define the FAScycle.

\section{(1) Presmoothing}

Compute $\left\{\bar{c}_{k}^{m}, \bar{\mu}_{k}^{m-\frac{1}{2}}\right\}$ by applying $\nu$ smoothing steps to $\left\{c_{k}^{m}, \mu_{k}^{m-\frac{1}{2}}\right\}$

$$
\left\{\bar{c}_{k}^{m}, \bar{\mu}_{k}^{m-\frac{1}{2}}\right\}=\operatorname{SMOOTH} H^{\nu}\left(c_{k}^{n}, c_{k}^{m}, \mu_{k}^{m-\frac{1}{2}}, \mathbf{N S O}_{k}, f_{k}^{n}, g_{k}^{n}\right),
$$

which means performing $\nu$ smoothing steps with initial approximation $c_{k}^{m}, \mu_{k}^{m-\frac{1}{2}}$, $c_{k}^{n}$, source terms $f_{k}^{n}, g_{k}^{n}$, and the $S M O O T H$ relaxation operator (see Appendix B for its derivation) to get the approximation $\bar{c}_{k}^{m}, \bar{\mu}_{k}^{m-\frac{1}{2}}$.

One $S M O O T H$ relaxation operator step consists of solving the system (34) and (35) given below by $2 \times 2$ matrix inversion for each $i$ and $j$.

$$
\frac{\bar{c}_{i j}^{m}}{\Delta t}+\left(\frac{2}{\Delta x^{2}}+\frac{2}{\Delta y^{2}}\right) \bar{\mu}_{i j}^{m-\frac{1}{2}}=\frac{\mu_{i+1, j}^{m-\frac{1}{2}}+\bar{\mu}_{i-1, j}^{m-\frac{1}{2}}}{\Delta x^{2}}+\frac{\mu_{i, j+1}^{m-\frac{1}{2}}+\bar{\mu}_{i, j-1}^{m-\frac{1}{2}}}{\Delta y^{2}}+f_{i j}^{n}
$$

and

$$
\begin{aligned}
-\left[\frac{\epsilon^{2}}{2}\left(\frac{2}{\Delta x^{2}}+\frac{2}{\Delta y^{2}}\right)+\right. & \left.\frac{\partial \hat{\phi}\left(c_{i j}^{n}, c_{i j}^{m}\right)}{\partial v}\right] \bar{c}_{i j}^{m}+\bar{\mu}_{i j}^{m-\frac{1}{2}} \\
= & g_{i j}^{n}+\hat{\phi}\left(c_{i j}^{n}, c_{i j}^{m}\right)-\frac{\partial \hat{\phi}\left(c_{i j}^{n}, c_{i j}^{m}\right)}{\partial v} c_{i j}^{m} \\
& -\frac{\epsilon^{2}}{2 \Delta x^{2}}\left(c_{i+1, j}^{m}+\bar{c}_{i-1, j}^{m}\right)-\frac{\epsilon^{2}}{2 \Delta y^{2}}\left(c_{i, j+1}^{m}+\bar{c}_{i, j-1}^{m}\right) .
\end{aligned}
$$

\section{(2) Compute the defect}




$$
\left(\bar{d}_{1 k}^{m}, \bar{d}_{2 k}^{m}\right)=\left(f_{k}^{n}, g_{k}^{n}\right)-\mathbf{N S O}_{k}\left(\bar{c}_{k}^{n}, \bar{c}_{k}^{m}, \bar{\mu}_{k}^{m-\frac{1}{2}}\right)
$$

(3) Restrict the defect and $\left\{\bar{c}_{k}^{m}, \bar{\mu}_{k}^{m-\frac{1}{2}}\right\}$

$$
\begin{aligned}
\left(\bar{d}_{1 k-1}^{m}, \bar{d}_{2 k-1}^{m}\right) & =I_{k}^{k-1}\left(\bar{d}_{1 k}^{m}, \bar{d}_{2 k}^{m}\right), \\
\left(\bar{c}_{k-1}^{m}, \bar{\mu}_{k-1}^{m-\frac{1}{2}}\right) & =I_{k}^{k-1}\left(\bar{c}_{k}^{m}, \bar{\mu}_{k}^{m-\frac{1}{2}}\right) .
\end{aligned}
$$

The restriction operator $I_{k}^{k-1}$ maps $k$-level functions to $(k-1)$-level functions.

$$
\begin{aligned}
d_{k-1}\left(x_{i}, y_{j}\right)=I_{k}^{k-1} d_{k}\left(x_{i}, y_{j}\right)= & \frac{1}{4}\left[d_{k}\left(x_{i}-h / 2, y_{j}-\frac{h}{2}\right)+d_{k}\left(x_{i}-h / 2, y_{j}+\frac{h}{2}\right)\right. \\
& \left.+d_{k}\left(x_{i}+h / 2, y_{j}-\frac{h}{2}\right)+d_{k}\left(x_{i}+h / 2, y_{j}+\frac{h}{2}\right)\right]
\end{aligned}
$$

That is, coarse grid values are obtained by averaging the four nearby fine grid values.

(4) Compute the right-hand side

$$
\left(f_{k-1}^{n}, g_{k-1}^{n}\right)=\left(\bar{d}_{1 k-1}^{m}, \bar{d}_{2 k-1}^{m}\right)+\mathbf{N S O}_{k-1}\left(\bar{c}_{k-1}^{n}, \bar{c}_{k-1}^{m}, \bar{\mu}_{k-1}^{m-\frac{1}{2}}\right)
$$

(5) Compute an approximate solution $\left\{\hat{c}_{k-1}^{m}, \hat{\mu}_{k-1}^{m-\frac{1}{2}}\right\}$ of the coarse grid equation on $\Omega_{k-1}$, i.e. solve

$$
\mathbf{N S O}_{k-1}\left(c_{k-1}^{n}, \hat{c}_{k-1}^{m}, \hat{\mu}_{k-1}^{m-\frac{1}{2}}\right)=\left(f_{k-1}^{n}, g_{k-1}^{n}\right) .
$$

If $k=1$, we explicitly invert a $2 \times 2$ matrix to obtain the solution. If $k>1$, we solve (36) by performing a FAS $k$-grid cycle using $\left\{\bar{c}_{k-1}^{m}, \bar{\mu}_{k-1}^{m-\frac{1}{2}}\right\}$ as an initial approximation:

$$
\left\{\hat{c}_{k-1}^{m}, \hat{\mu}_{k-1}^{m-\frac{1}{2}}\right\}=\operatorname{FAScycle}\left(k-1, c_{k-1}^{n}, \bar{c}_{k-1}^{m}, \bar{\mu}_{k-1}^{m-\frac{1}{2}}, \mathbf{N S O}_{k-1}, f_{k-1}^{n}, g_{k-1}^{n}, \nu\right) .
$$

(6) Compute the coarse grid correction (CGC):

$$
\hat{v}_{1 k-1}^{m}=\hat{c}_{k-1}^{m}-\bar{c}_{k-1}^{m} \text {. }
$$




$$
{\hat{v_{2 k-1}}}_{2 m-\frac{1}{2}}^{m}=\hat{\mu}_{k-1}^{m-\frac{1}{2}}-\bar{\mu}_{k-1}^{m-\frac{1}{2}}
$$

\section{(7) Interpolate the correction}

$$
\begin{gathered}
\hat{v}_{1 k}^{m}=I_{k-1}^{k} \hat{v}_{1 k-1}^{m} . \\
\hat{v}_{2 k}^{m-\frac{1}{2}}=I_{k-1}^{k} \hat{v}_{2 k-1}^{m-\frac{1}{2}} .
\end{gathered}
$$

The interpolation operator $I_{k-1}^{k}$ maps (k-1)-level functions to k-level functions. Here, the coarse values are simply transferred to the four nearby fine grid points, i.e. $v_{k}\left(x_{i}, y_{j}\right)=I_{k-1}^{k} v_{k-1}\left(x_{i}, y_{j}\right)=v_{k-1}\left(x_{i}+\frac{h}{2}, y_{j}+\frac{h}{2}\right)$ for $i$ and $j$ odd-numbered integers. The values at the other node points are given by

$$
v_{k}\left(x_{i}+h, y_{j}\right)=v_{k}\left(x_{i}, y_{j}+h\right)=v_{k}\left(x_{i}+h, y_{j}+h\right)=v_{k-1}\left(x_{i}+\frac{h}{2}, y_{j}+\frac{h}{2}\right),
$$

where $i$ and $j$ are odd.

(8) Compute the corrected approximation on $\Omega_{k}$

$$
\begin{gathered}
c_{k}^{m, \text { after } C G C}=\bar{c}_{k}^{m}+\hat{v}_{1 k}^{m} . \\
\mu_{k}^{m-\frac{1}{2}, \text { after } C G C}=\bar{\mu}_{k}^{m-\frac{1}{2}}+\hat{v}_{2 k}^{m-\frac{1}{2}} .
\end{gathered}
$$

(9) Postsmoothing Compute $\left\{c_{k}^{m+1}, \mu_{k}^{m+\frac{1}{2}}\right\}$ by applying $\nu$ smoothing steps to $c_{k}^{m}$, after $C G C$, $\mu_{k}^{m-\frac{1}{2}}$, after $C G C$

$\left\{c_{k}^{m+1}, \mu_{k}^{m+\frac{1}{2}}\right\}=S \operatorname{MOOTH} H^{\nu}\left(c_{k}^{n}, c_{k}^{m, \text { after } C G C}, \mu_{k}^{m-\frac{1}{2}}\right.$, after $\left.C G C, \mathbf{N S O}_{k}, f_{k}^{n}, g_{k}^{n}\right)$

This completes the description of a nonlinear FAScycle. We next turn to the solution of the discrete system in the presence of flow.

\section{Solution of system in presence of flow}

Here, we present an iterative projection method for solving the coupled discrete NSCH system (14), (17) and (18)- (19). For simplicity, we focus on the case in which $\eta$ is constant. Let $u^{*, k+1}$ be an intermediate velocity field and satisfy the following system for the iteration number $k \geq 0$ : 


$$
\begin{aligned}
\frac{\mathbf{u}^{*, k+1}-\mathbf{u}^{n}}{\Delta t}+ & \omega^{n+\frac{1}{2}, k} \times \mathbf{u}^{n+\frac{1}{2}, k}=-\tilde{\nabla}_{d}^{c} P^{n+\frac{1}{2}, k}+\delta^{k} \mathbf{u}^{n+\frac{1}{2}, k} \\
& +\frac{1}{2 \mathbf{R e}} \Delta_{d}\left(\mathbf{u}^{*, k+1}+\mathbf{u}^{n}\right)-\frac{\mathbf{W e}_{s}^{-1}}{\epsilon} c^{n+\frac{1}{2}, k} \nabla_{d} \mu^{n+\frac{1}{2}, k}
\end{aligned}
$$

where

$$
\begin{aligned}
& \omega_{i j}^{n+\frac{1}{2}, k} \times \mathbf{u}_{i j}^{n+\frac{1}{2}, k}= \\
& \left(-\frac{\bar{v}_{i+1, j}-\bar{v}_{i-1, j}-\bar{u}_{i, j+1}+\bar{u}_{i, j-1}}{2 h} \bar{v}_{i j}, \frac{\bar{v}_{i+1, j}-\bar{v}_{i-1, j}-\bar{u}_{i, j+1}+\bar{u}_{i, j-1}}{2 h} \bar{u}_{i j}\right),
\end{aligned}
$$

and

$$
\delta^{k}=\left(\mathbf{u}^{n+\frac{1}{2}, k}, \nabla_{d}^{c} P^{n+\frac{1}{2}, k}\right)_{h} /\left\|\mathbf{u}^{n+\frac{1}{2}, k}\right\|^{2}
$$

and $(\bar{u}, \bar{v})=\mathbf{u}^{n+\frac{1}{2}, k} \equiv\left(\mathbf{u}^{n+1, k}+\mathbf{u}^{n}\right) / 2$ and $c^{n+\frac{1}{2}, k}$ is defined analogously. In the first step of the iteration, we take $\mathbf{u}^{n+1,0}=\mathbf{u}^{n}$ and $p^{n+\frac{1}{2}, 0}=p^{n-\frac{1}{2}}$. Note that the pressure gradient, advection and surface force terms are treated as forcing functions. The fact that the latter two terms are treated in this way ensures, from lemma 6 , that the discrete system has an energy functional as $k \rightarrow \infty$, assuming that the iteration converges. Numerically, we found convergence is typically achieved in just two or three iterations. We are also investigating other algorithms for which the $\mathbf{C H}$ and NS equations are solved simultaneously. Following [1,2,55], Eq. (37) is solved for $\mathbf{u}^{*, k+1}$ using a linear multigrid method. For completeness, this scheme is given in Appendix C.

The velocity field $\mathbf{u}^{*, k+1}$ is not, in general, divergence-free. The projection step of the algorithm decomposes the intermediate velocity into a discrete gradient of a scalar potential and an approximately divergence-free vector field. They correspond to the pressure gradient and to the velocity updates, respectively. In particular, if $\mathcal{P}$ represents the projection operator, then

$$
\frac{\mathbf{u}^{n+1, k+1}-\mathbf{u}^{n}}{\Delta t}=\mathcal{P}\left(\frac{\mathbf{u}^{*, k+1}-\mathbf{u}^{n}}{\Delta t}\right) \equiv \frac{\mathbf{u}^{*, k+1}-\mathbf{u}^{n}}{\Delta t}-\tilde{\nabla}_{d}^{c} \psi
$$

where $\psi$ is obtained by

$$
\Delta_{d}^{c} \psi=\nabla_{d}^{c} \cdot\left(\frac{\mathbf{u}^{*, k+1}-\mathbf{u}^{n}}{\Delta t}\right)
$$

with Neumann boundary conditions which are imposed by introducing a ring of ghost-cells surrounding the physical domain. Note that the velocity $\mathbf{u}^{n+1, k+1}$ is only approximately divergence-free, i.e.

$$
\nabla_{d}^{c} \cdot\left(\frac{\mathbf{u}^{n+1, k+1}-\mathbf{u}^{n}}{\Delta t}\right)=\left(\Delta_{d}^{c}-\nabla_{d}^{c} \cdot \tilde{\nabla}_{d}^{c}\right) \psi
$$


The pressure is then updated using the second-order accurate scheme [12]:

$$
p^{n+\frac{1}{2}, k+1}=p^{n+\frac{1}{2}, k}+\psi-\frac{\Delta t}{2 \mathbf{R e}} \Delta_{d}^{c} \psi
$$

We note that combining Eqs. (42) and (41) and letting $k \rightarrow \infty$ (assuming that the iteration converges) yields Eq. (18) as claimed in section 2. Analogously, plugging Eq. (39) into Eq. (37), and using Eq. (42), yields Eq. (19) also as claimed.

The approximate projection Poisson's Eq. (40) is solved using a linear multigrid method. Similar multigrid methods have been used in 3D [2] and in a finite element context in 2D [1]. The multigrid algorithm we use is analogous to that presented in Appendix $\mathrm{C}$ for the intermediate velocity. In the case of approximate projection, we use the Gauss-Seidel smoothing operator on grid level $k$, i.e. $\bar{\psi}_{k}^{m}=S \operatorname{MOOTH} H^{\nu}\left(\psi_{k}^{m}, L_{k}, f_{k}\right)$ where $L_{k}$ is the Laplacian operator $\Delta_{d}^{c}$ on the $k$ grid level. The smoothing operation is

$$
\bar{\psi}_{i+\frac{1}{2}, j+\frac{1}{2}}^{m}=\frac{1}{4}\left(\bar{\psi}_{i-\frac{1}{2}, j+\frac{1}{2}}^{m}+\psi_{i+\frac{1}{2}, j+\frac{3}{2}}^{m}+\bar{\psi}_{i+\frac{1}{2}, j-\frac{1}{2}}^{m}+\psi_{i+\frac{3}{2}, j+\frac{1}{2}}^{m}\right)-\frac{h^{2}}{4} f_{i+\frac{1}{2}, j+\frac{1}{2}}
$$

where the forcing term is

$$
f_{i+\frac{1}{2}, j+\frac{1}{2}}=\nabla_{d}^{c} \cdot\left(\frac{\mathbf{u}_{i j}^{*, k+1}-\mathbf{u}_{i j}^{n}}{\Delta t}\right)
$$

In section 5, we analyze the projection algorithm and demonstrate that the multigrid method convergence rate is insensitive to the grid size.

We next update the concentration field by

$$
\begin{gathered}
\frac{c^{n+1, k+1}-c^{n}}{\Delta t}+\nabla_{d} \cdot\left(\mathbf{u}^{n+\frac{1}{2}, k+1} c^{n+\frac{1}{2}, k}\right)=\Delta_{d} \mu^{n+\frac{1}{2}, k+1}, \\
\mu^{n+\frac{1}{2}, k+1}=\hat{\phi}\left(c^{n}, c^{n+1, k+1}\right)-\frac{\epsilon^{2}}{2} \Delta_{d}\left(c^{n}+c^{n+1, k+1}\right) .
\end{gathered}
$$

This system is solved using the non-linear multigrid method presented in section 3 where the source term $f^{n}$ is modified to account for the advection.

The above system (37)-(39) and (43)-(44) is iterated in $k$ until the difference between successive iterates is less than an error tolerance. As mentioned above, typically only two or three iterations are required to achieve an error tolerance of $10^{-7}$. We found that this number is insensitive to the value of the Weber number. 


\section{Local Fourier Analysis}

\subsection{Absence of flow}

To analyze the behavior of the multigrid method, we linearize the nonlinear scheme and perform a local Fourier analysis (e.g. see [55]). In particular, we analyze the smoother since the performance of the multigrid method depends strongly on the smoother.

Let $c^{n+1}, \mu^{n+1 / 2}$ be the solution of

$$
\begin{aligned}
& \frac{c_{i j}^{n+1}-c_{i j}^{n}}{\Delta t}=\Delta_{d} \mu_{i j}^{n+\frac{1}{2}}, \\
& \mu_{i j}^{n+\frac{1}{2}}=\hat{\phi}\left(c_{i j}^{n}, c_{i j}^{n+1}\right)-\frac{\epsilon^{2}}{2} \Delta_{d}\left(c_{i j}^{n+1}+c_{i j}^{n}\right) .
\end{aligned}
$$

After linearizing the nonlinear term $\hat{\phi}\left(c_{i j}^{n}, c_{i j}^{n+1}\right)=\frac{\alpha}{2}\left(c_{i j}^{n}+c_{i j}^{n+1}\right)+\beta$, where $\alpha=$ $\phi^{\prime}\left(c_{m}\right), c_{m}$ is an average concentration, and $\beta$ is a constant and substituting $\mu_{i j}^{n+\frac{1}{2}}$ into (45), the scheme becomes

$$
L_{h} c_{h}^{n+1}=f_{h}^{n}
$$

where

$$
\begin{aligned}
L_{h} c_{h}^{n+1}:= & \frac{c_{i j}^{n+1}}{\Delta t}-\frac{\alpha}{2 h^{2}}\left(c_{i-1, j}^{n+1}+c_{i, j-1}^{n+1}-4 c_{i j}^{n+1}\right) \\
& +\frac{\epsilon^{2}}{2 h^{4}}\left[c_{i-2, j}^{n+1}+c_{i, j-2}^{n+1}+2\left(c_{i-1, j+1}^{n+1}+c_{i-1, j-1}^{n+1}\right)\right. \\
& \left.-8\left(c_{i-1, j}^{n+1}+c_{i, j-1}^{n+1}\right)+20 c_{i, j}^{n+1}\right]-\frac{\alpha}{2 h^{2}}\left(c_{i+1, j}^{n+1}+c_{i, j+1}^{n+1}\right) \\
& +\frac{\epsilon^{2}}{2 h^{4}}\left[c_{i+2, j}^{n+1}+c_{i, j+2}^{n+1}+2\left(c_{i+1, j+1}^{n+1}+c_{i+1, j-1}^{n+1}\right)-8\left(c_{i+1, j}^{n+1}+c_{i, j+1}^{n+1}\right)\right]
\end{aligned}
$$

and

$$
f_{h}^{n}=\frac{\alpha}{2} \Delta_{d} c_{i j}^{n}-\frac{\epsilon^{2}}{2} \Delta_{d}^{2} c_{i j}^{n}+\frac{c_{i j}^{n}}{\Delta t} .
$$

For Gauss-Seidel iteration with a lexicographic ordering of the grid points applied to the above equation, we have the following operator decomposition. 


$$
\begin{aligned}
L_{h}^{+} c_{h}^{n+1}:= & \frac{c_{i j}^{n+1}}{\Delta t}-\frac{\alpha}{2 h^{2}}\left(c_{i-1, j}^{n+1}+c_{i, j-1}^{n+1}-4 c_{i j}^{n+1}\right)+\frac{\epsilon^{2}}{2 h^{4}}\left[c_{i-2, j}^{n+1}+c_{i, j-2}^{n+1}\right. \\
& \left.+2\left(c_{i-1, j+1}^{n+1}+c_{i-1, j-1}^{n+1}\right)-8\left(c_{i-1, j}^{n+1}+c_{i, j-1}^{n+1}\right)+20 c_{i, j}^{n+1}\right], \\
L_{h}^{-} c_{h}^{n+1}:= & -\frac{\alpha}{2 h^{2}}\left(c_{i+1, j}^{n+1}+c_{i, j+1}^{n+1}\right)+\frac{\epsilon^{2}}{2 h^{4}}\left[c_{i+2, j}^{n+1}+c_{i, j+2}^{n+1}+2\left(c_{i+1, j+1}^{n+1}+c_{i+1, j-1}^{n+1}\right)\right. \\
& \left.-8\left(c_{i+1, j}^{n+1}+c_{i, j+1}^{n+1}\right)\right] .
\end{aligned}
$$

Therefore, this relaxation method (which is the linear analogue of the nonlinear multigrid smoother) can be written locally as

$$
L_{h}^{+} \tilde{z}_{h}+L_{h}^{-} z_{h}=f_{h}^{n}
$$

where $z_{h}$ corresponds to the old approximation of $c_{h}$ (approximation before the relaxation step) and $\tilde{z}_{h}$ to the new approximation (after the step). Subtracting (47) from the discrete equation $L_{h} c_{h}=f_{h}$ and letting $\tilde{v}_{h}=c_{h}-\tilde{z}_{h}$ and $v_{h}=c_{h}-z_{h}$, we obtain the equation

$$
L_{h}^{+} \tilde{v}_{h}+L_{h}^{-} v_{h}=0
$$

or, equivalently,

$$
\tilde{v}_{h}=S_{h} v_{h}
$$

where $S_{h}=-\left(L_{h}^{+}\right)^{-1} L_{h}^{-}$is the resulting smoothing operator. Applying $L_{h}^{+}$and $L_{h}^{-}$ to the formal eigenfunctions $e^{i \theta_{1} x / h} e^{i \theta_{2} y / h}$, we obtain

$$
\begin{aligned}
& L_{h}^{+} e^{i \theta_{1} x / h} e^{i \theta_{2} y / h}=\hat{L}_{h}^{+} e^{i \theta_{1} x / h} e^{i \theta_{2} y / h}, \\
& L_{h}^{-} e^{i \theta_{1} x / h} e^{i \theta_{2} y / h}=\hat{L}_{h}^{-} e^{i \theta_{1} x / h} e^{i \theta_{2} y / h},
\end{aligned}
$$

where $\hat{L}_{h}^{+}$and $\hat{L}_{h}^{-}$are the symbols of the operators $L_{h}^{+}$and $L_{h}^{-}$, respectively:

$$
\begin{aligned}
\hat{L}_{h}^{+}\left(\theta_{1}, \theta_{2}\right)= & \frac{1}{\Delta t}-\frac{\alpha}{2 h^{2}}\left(e^{-i \theta_{1}}+e^{-i \theta_{2}}-4\right)+\frac{\epsilon^{2}}{2 h^{4}}\left[e^{-2 i \theta_{1}}+e^{-2 i \theta_{2}}\right. \\
& \left.+2\left(e^{-i\left(\theta_{1}-\theta_{2}\right)}+e^{-i\left(\theta_{1}+\theta_{2}\right)}\right)-8\left(e^{-i \theta_{1}}+e^{-i \theta_{2}}\right)+20\right], \\
\hat{L}_{h}^{-}\left(\theta_{1}, \theta_{2}\right)= & -\frac{\alpha}{2 h^{2}}\left(e^{i \theta_{1}}+e^{i \theta_{2}}\right)+\frac{\epsilon^{2}}{2 h^{4}}\left[e^{2 i \theta_{1}}+e^{2 i \theta_{2}}\right. \\
& \left.+2\left(e^{i\left(\theta_{1}+\theta_{2}\right)}+e^{i\left(\theta_{1}-\theta_{2}\right)}\right)-8\left(e^{i \theta_{1}}+e^{i \theta_{2}}\right)\right] .
\end{aligned}
$$

The amplification factor of the relaxation scheme is

$$
\hat{S}_{h}\left(\theta_{1}, \theta_{2}\right):=-\frac{\hat{L}_{h}^{-}\left(\theta_{1}, \theta_{2}\right)}{\hat{L}_{h}^{+}\left(\theta_{1}, \theta_{2}\right)} .
$$

Define the high frequency smoothing (HFS) factor:

$$
\mu_{l o c}\left(S_{h}\right):=\sup \left\{\left|\hat{S}_{h}\left(\theta_{1}, \theta_{2}\right)\right|: \frac{\pi}{2} \leq\left|\theta_{1}\right|,\left|\theta_{2}\right| \leq \pi\right\}
$$


Here, as is typically done [55], we assume that the coarse grid operations are ideal and annihilate the low frequency error components while leaving the high frequency components unchanged. Therefore, we only consider $\frac{\pi}{2} \leq\left|\theta_{1}\right|,\left|\theta_{2}\right| \leq \pi$. We define a convergence factor as an average of the quantity $\left\|d_{h}^{m}\right\| /\left\|d_{h}^{m-1}\right\|$, where $d_{h}^{m}(m=1,2, \cdots)$ are the defects.

The convergence factor is estimated numerically using our nonlinear code with the parameters $\epsilon=0.01$, and the mesh-dependent time step $\Delta t=0.1 \mathrm{~h}$ and initial conditions

$$
c_{0}(x, y)= \begin{cases}0.0+0.01 \cos (0.5 \pi x / h) \cos (0.5 \pi y / h), & \alpha=1 / 4 \\ 0.5+0.01 \cos (0.5 \pi x / h) \cos (0.5 \pi y / h), & \alpha=-1 / 4\end{cases}
$$

We measure the $V(m, n)$ - HFS factors, where $m$ and $n$ are the numbers of presmoothing and post-smoothing, with different mesh sizes. We focus on $m=1$ and $n=0$ and 1 as these yield the most efficient algorithms. In addition, we consider $\alpha= \pm 1 / 4$ where the positive (negative) values correspond to linearization in the stable (unstable, i.e. spinodal region) ranges of the evolution.

Table 1 shows HFS factors and measured V(m,n)-cycle convergence factors with different mesh sizes and $\alpha=0.25$. Note that $\sqrt{V(1,1)}$-cycle means the square root of $\mathrm{V}(1,1)$-cycle convergence factor. Observe that with $m=1$ and $n=0$, the HFS factor tends to 1 as the mesh is refined and thus suggests that the number of $\mathrm{V}$ cycles required to solve the full system increases with increasing resolution. In fact this result is comparable to simply using Gauss-Siedel iteration without multigrid. However, the HFS factor corresponding to $m=1$ and $n=1$ remains uniformly bounded below 1 with increasing resolution and apparently converges to 0.4196 as $h \rightarrow 0$. This is significantly below the theoretical estimate 0.6694 . Thus the number of $V(1,1)$-cycles required to solve the full problem is insensitive to the resolution.

The HFS factors for $\alpha=-0.25$ are given in Table 2. Their behavior is analogous to that observed for the $\alpha=0.25$. Note that the HFS factor for $V(1,0)$-cycles is actually greater than 1 at the finest resolution.

Table 1. HFS factors for different mesh sizes. $\alpha=0.25, \Delta t=0.1 h$ and $h=1 / N$ 


\begin{tabular}{||c|c|c|c|c||}
\hline Case & $16 \times 16$ & $32 \times 32$ & $64 \times 64$ & $128 \times 128$ \\
\hline$\mu_{\text {loc }}$ & 0.0363 & 0.2839 & 0.5460 & 0.6376 \\
\hline $\mathrm{V}(1,0)$-cycle & 0.1643 & 0.2108 & 0.2865 & 0.3489 \\
\hline$\sqrt{V(1,1)}$-cycle & 0.2108 & 0.2462 & 0.2840 & 0.3434 \\
\hline \hline Case & $256 \times 256$ & $512 \times 512$ & $1024 \times 1024$ & $2048 \times 2048$ \\
\hline$\mu_{\text {loc }}$ & 0.6616 & 0.6676 & 0.6690 & 0.6694 \\
\hline $\mathrm{V}(1,0)$-cycle & 0.4636 & 0.6563 & 0.8198 & 0.9810 \\
\hline$\sqrt{V(1,1)}$-cycle & 0.4021 & 0.4171 & 0.4196 & 0.4196 \\
\hline
\end{tabular}

Table 2. HFS factors for different mesh sizes. $\alpha=-0.25, \Delta t=0.1 h$ and $h=1 / N$

\begin{tabular}{||c|c|c|c|c||}
\hline Case & $16 \times 16$ & $32 \times 32$ & $64 \times 64$ & $128 \times 128$ \\
\hline$\mu_{\text {loc }}$ & 1.3773 & 1.1563 & 0.7874 & 0.6992 \\
\hline $\mathrm{V}(1,0)$-cycle & 0.5183 & 0.5837 & 0.4245 & 0.4829 \\
\hline$\sqrt{V(1,1)}$-cycle & 0.5501 & 0.6061 & 0.3706 & 0.4162 \\
\hline \hline Case & $256 \times 256$ & $512 \times 512$ & $1024 \times 1024$ & $2048 \times 2048$ \\
\hline$\mu_{\text {loc }}$ & 0.6771 & 0.6714 & 0.6700 & 0.6697 \\
\hline $\mathrm{V}(1,0)$-cycle & 0.6260 & 0.7904 & 0.8719 & N/A \\
\hline$\sqrt{V(1,1)}$-cycle & 0.4141 & 0.4209 & 0.4299 & 0.4382 \\
\hline
\end{tabular}

Together, these results for $\alpha= \pm 0.25$ suggest that the multigrid method using a $V(1,1)$-cycle with time step $\Delta t \sim h$ converges uniformly with respect to increasing resolution. Correspondingly, this would impose a first order time step constraint on our discrete scheme to solve the $\mathbf{C H}$ equation.

Next, let us consider results obtained using a fixed $\Delta t=0.005$ independent of the mesh size $h$. All other parameters are as before. This value of $\Delta t$ roughly corresponds to that used in Tables 1 and 2 with $h=1 / 16$. Table 3 shows the $V(1,1)$-HFS factors with different mesh sizes and $\alpha=0.25$. Again, the results are qualitatively similar to the case with variable $\Delta t \sim h$. When $\alpha=-0.25$, however, the results are very different. This is seen in Table 4 . The numerical HFS factor now tends to 
1 as the mesh is refined in spite of the fact that theoretical HFS factors are comparable for all choices of $\Delta t$ and $\alpha$. This behavior at fine grids occurs because the coarse grid correction steps internal to the $V$-cycle use much larger time steps when $\Delta t$ is fixed than when $\Delta t \sim h$ where $h$ is the fine grid size. This can be seen in figs. 1(left,right) where diagonal slices of the amplification factors $\left|\hat{S}_{h}(\theta, \theta)\right|$, for $\theta \in[-\pi, \pi]$, are shown for $h=1 / 16,1 / 32$ and $1 / 2048$ where $\Delta t=0.005$ (left) and $\Delta t=0.1 / 2048$ (right). Observe that for the latter case, the amplification factors for the coarse meshes are nearly equal to zero in contrast to the case with the larger time step.

These results suggest that when a fixed $\Delta t$ is used, it's value should be such that the coarse grid HFS factors are less than 1 . This is confirmed in Table 5 where the HFS factors are shown with $\Delta t=0.1 / 64=1.5625 \times 10^{-3}$. This suggests that the multigrid method using a $V(1,1)$-cycle with fixed time step $\Delta t$ converges uniformly with respect to increasing resolution if $\Delta t$ is small enough. This would impose no grid-dependent time step constraints on our discrete scheme to solve the CH equation.

Table 3. HFS factors for different mesh sizes. $\alpha=0.25$ and $\Delta t=5 \times 10^{-3}$

\begin{tabular}{||c|c|c|c|c||}
\hline Case & $16 \times 16$ & $32 \times 32$ & $64 \times 64$ & $128 \times 128$ \\
\hline$\mu_{\text {loc }}$ & 0.0329 & 0.2984 & 0.5542 & 0.6392 \\
\hline$\sqrt{V(1,1)}$-cycle & 0.1930 & 0.2706 & 0.3428 & 0.3920 \\
\hline \hline Case & $256 \times 256$ & $512 \times 512$ & $1024 \times 1024$ & $2048 \times 2048$ \\
\hline$\mu_{\text {loc }}$ & 0.6618 & 0.6676 & 0.6691 & 0.6694 \\
\hline$\sqrt{V(1,1)}$-cycle & 0.4127 & 0.4199 & 0.4294 & 0.4452 \\
\hline
\end{tabular}

Table 4. HFS factors for different mesh sizes. $\alpha=-0.25$ and $\Delta t=5 \times 10^{-3}$

\begin{tabular}{||c|c|c|c|c||}
\hline Case & $16 \times 16$ & $32 \times 32$ & $64 \times 64$ & $128 \times 128$ \\
\hline$\mu_{l o c}$ & 0.9594 & 1.2796 & 0.8014 & 0.7009 \\
\hline$\sqrt{V(1,1)}$-cycle & 0.3925 & 0.8503 & 0.9098 & 0.9177 \\
\hline \hline Case & $256 \times 256$ & $512 \times 512$ & $1024 \times 1024$ & $2048 \times 2048$ \\
\hline$\mu_{l o c}$ & 0.6773 & 0.6715 & 0.6700 & 0.6697 \\
\hline$\sqrt{V(1,1)}$-cycle & 0.9209 & 0.9220 & 0.9208 & 0.9860 \\
\hline
\end{tabular}


Table 5. HFS factors for different mesh sizes. $\alpha=-0.25, \Delta t=.1 / 64=1.5625 \times 10^{-3}$

\begin{tabular}{||c|c|c|c|c||}
\hline Case & $16 \times 16$ & $32 \times 32$ & $64 \times 64$ & $128 \times 128$ \\
\hline$\mu_{l o c}$ & 0.2324 & 0.9016 & 0.7874 & 0.7002 \\
\hline$\sqrt{V(1,1)}$-cycle & 0.1305 & 0.5117 & 0.3522 & 0.3907 \\
\hline \hline Case & $256 \times 256$ & $512 \times 512$ & $1024 \times 1024$ & $2048 \times 2048$ \\
\hline$\mu_{l o c}$ & 0.6772 & 0.6715 & 0.6700 & 0.6697 \\
\hline$\sqrt{V(1,1)}-$ cycle & 0.4228 & 0.4206 & 0.4290 & 0.4495 \\
\hline
\end{tabular}
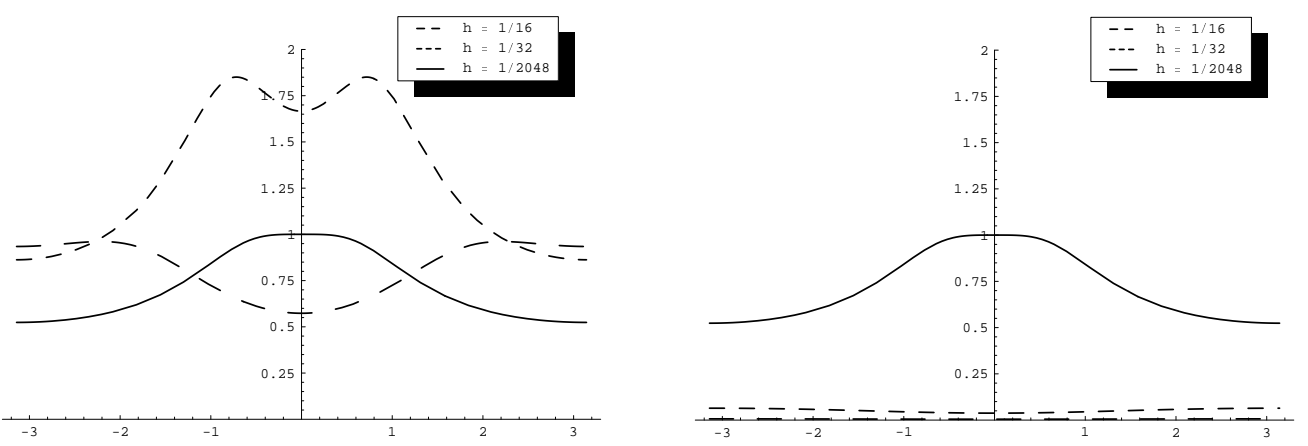

Fig. 1. Diagonal slice of amplification factor $\left|\hat{S}_{h}(\theta, \theta)\right|$, over the interval $[-\pi, \pi]$. In the left figure, $\Delta t=0.005$. In the right figure, $\Delta t=0.1 / 2048$.

\subsection{Presence of flow}

Here, we analyze approximate projection algorithm given in section 4 (an analysis of the multigrid method used to solve for the intermediate velocity is similar and thus is not presented). The multigrid smoothing operator $L_{h}^{a p p r o x}=L_{h}^{a,+}+L_{h}^{a,-}$ where

$$
\begin{aligned}
& L_{h}^{a,+} \psi:=\left(\psi_{i+\frac{1}{2}, j+\frac{3}{2}}-4 \psi_{i+\frac{1}{2}, j+\frac{1}{2}}+\psi_{i+\frac{3}{2}, j+\frac{1}{2}}\right) / h^{2} \\
& L_{h}^{a,-} \psi:=\left(\psi_{i-\frac{1}{2}, j+\frac{1}{2}}+\psi_{i+\frac{1}{2}, j-\frac{1}{2}}\right) / h^{2} .
\end{aligned}
$$

The resulting amplification factor is

$$
\hat{S}_{h}^{a}\left(\theta_{1}, \theta_{2}\right):=-\frac{\hat{L}_{h}^{a,-}\left(\theta_{1}, \theta_{2}\right)}{\hat{L}_{h}^{a,+}\left(\theta_{1}, \theta_{2}\right)},
$$


where the symbols are calculated as in section 5.1. In Fig. 2 (left), the diagonal slice $\left|\hat{S}_{h}^{a}(\theta, \theta)\right|$ is shown. Observe that the HFS factor $\mu_{l o c} \approx 0.45$ which suggests that the linear multigrid converges uniformly with respect to increasing resolution. For purposes of comparison, the corresponding amplification factor is shown (right) for the exact projection method (i.e. where $\Delta_{d}^{c} \equiv \nabla_{d}^{c} \cdot \tilde{\nabla}_{d}^{c}$ ). In the case of exact projection, however, the HFS factor $\mu_{l o c} \approx 1.0$ and suggests that the smoothing operator does not remove high frequencies efficiently which reflects the ill-conditioning of the system. The corresponding multigrid method requires a prohibitive number of iterations to converge when the mesh size $h$ is small. This is why the approximate projection algorithm is used (see also [45]).
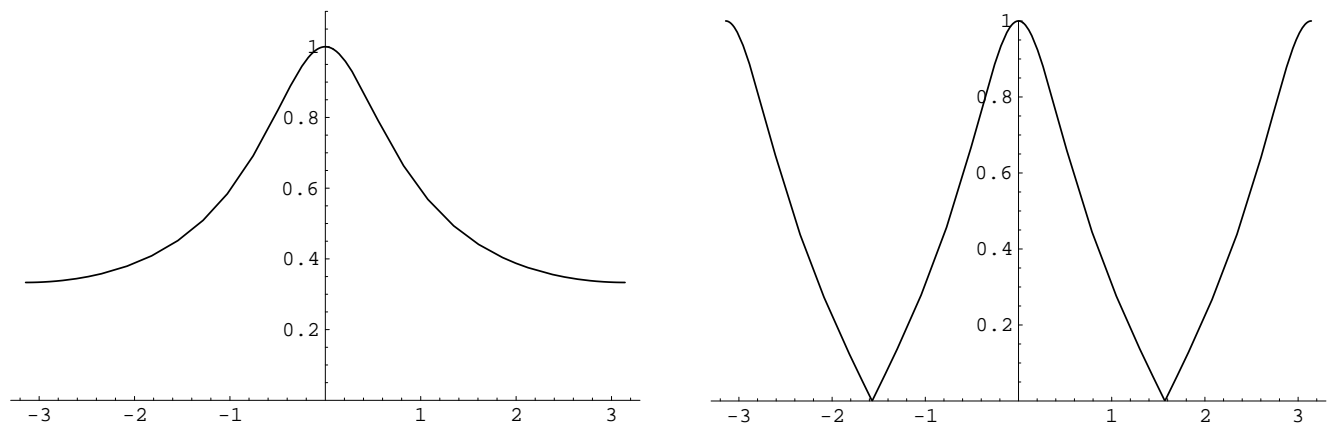

Fig. 2. Diagonal slices of amplification factor. Approximate projection (left), exact projection (right).

\section{Numerical experiments}

In this section, we validate our scheme by verifying the second-order convergence and comparing the numerical results with the prediction of a linear stability analysis. We then perform simulations of spinodal decomposition and examine the effect of boundary conditions, flow and interfacial tension.

\subsection{Convergence Test}

To obtain an estimate of the rate of convergence, we perform a number of simulations for a sample initial problem on a set of increasingly finer grids. We begin by considering the case in the absence of flow $(\mathbf{u}=0)$. The initial data is

$$
c_{0}(x, y)=0.5+0.12 \cos (2 \pi x) \cos (2 \pi y)+0.2 \cos (\pi x) \cos (3 \pi y)
$$

on a square domain, $[0,1] \times[0,1]$. The numerical solutions are computed on the uniform grids, $\Delta x=\Delta y=h=1 / 2^{n}$ for $n=4,5,6,7,8,9,10$, and 11. For each case, the convergence is measured at time $t=0.2$, the uniform time steps, $\Delta t=0.1 h$ and $\epsilon=0.01$, are used to establish the convergence rates. 


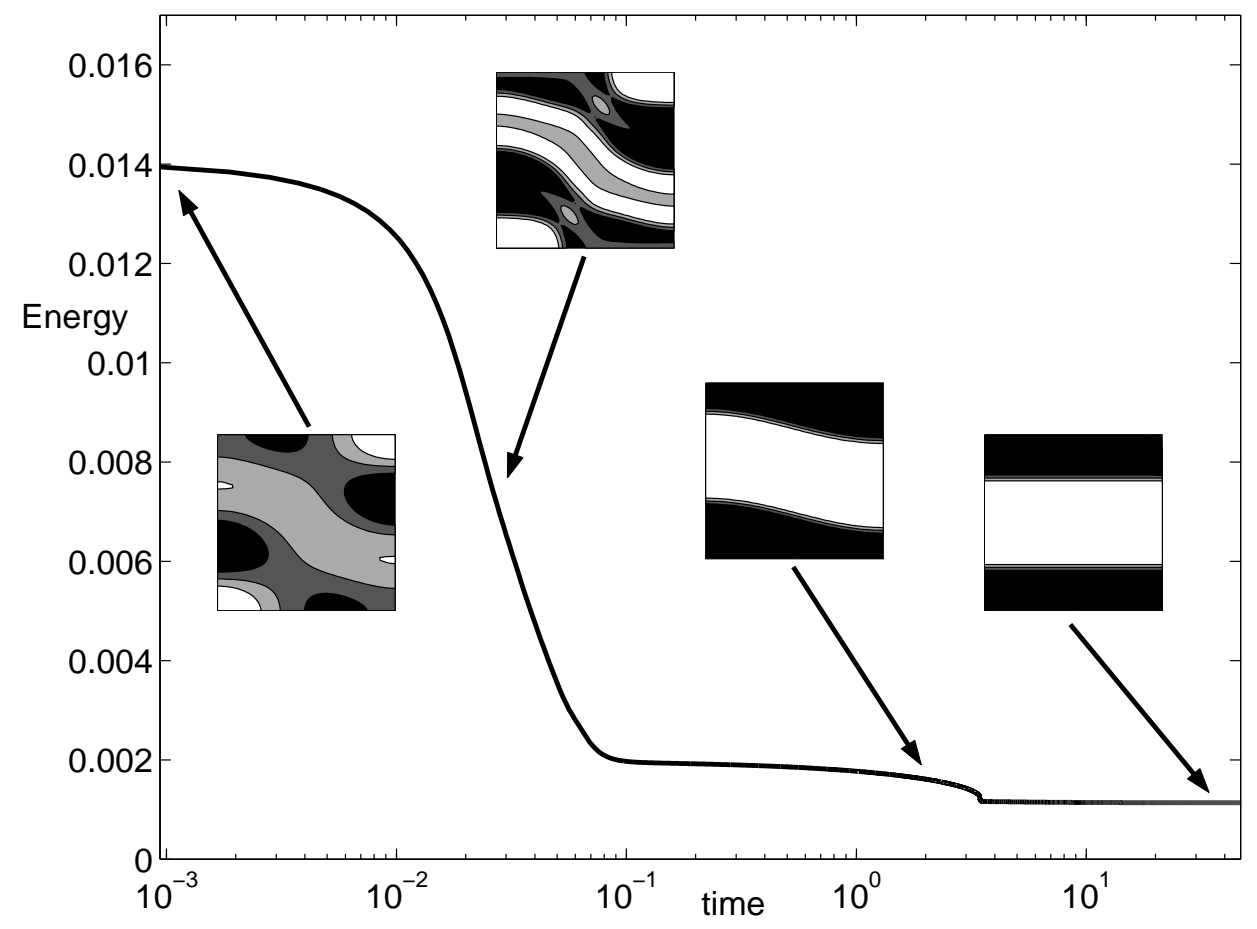

Fig. 3. The time dependent energy of the numerical solutions with the initial data (48). Snapshots of the concentation field are shown with filled contours at the three levels $c=0.25,0.5$ and 0.75 .

In our formulation of the method for the $\mathbf{C H}$ equation, since a cell centered grid is used, we define the error to be the discrete $l_{2}$-norm of the difference between that grid and the average of the next finer grid cells covering it:

$$
e_{h / \frac{h}{2} i j} \stackrel{d e f}{=} c_{h i j}-\left(c_{\frac{h}{2}}{ }_{2 i, 2 j}+c_{\frac{h}{2}}{ }_{2 i-1,2 j}+c_{\frac{h}{2}}{ }_{2 i, 2 j-1}+c_{\frac{h}{2}}{ }_{2 i-1,2 j-1}\right) / 4 .
$$

The rate of convergence is defined as the ratio of successive errors:

$$
\log _{2}\left(\left\|e_{h / \frac{h}{2}}\right\| /\left\|e_{\frac{h}{2} / \frac{h}{4}}\right\|\right)
$$

The errors and rates of convergence are given in table 6 . The results suggest that the scheme is indeed second order accurate. The deterioration of the rates from 2 at higher resolutions is believed to be due to accumulation of errors from coarse grid correction steps internal to the nonlinear multigrid method. In figure 3 , the time evolution of the energy is shown accompanied with grey-scale contour images of the numerical solution $c$ at the (filled) levels from 0 to 0.25 (black), 0.25 to 0.5 , 0.5 to 0.75 and 0.75 to 1.0 (white). As expected from lemma 26, the energy is nonincreasing and tends to a constant value. The concentration phase-separates and depletes the center region of the domain. The phase accumuates at the $y$-boundaries which then straighten to lower the energy and to subsequently form two horizontal bands. This is in fact a local equilibrium for Neumann boundary conditions. The global equilibrium consists of a single interface. 
Table 6. $l_{2}$-norm of the Errors and Convergence rates for concentration $c$.

\begin{tabular}{||c|c|c|c|c|c|}
\hline Case & $16-32$ & Rate & $32-64$ & Rate & $64-128$ \\
\hline$l_{2}$ & $1.721 \mathrm{e}-01$ & $2.095 \mathrm{e}+00$ & $4.027 \mathrm{e}-02$ & $3.290 \mathrm{e}+00$ & $4.118 \mathrm{e}-03$ \\
\hline
\end{tabular}

\begin{tabular}{||c|c|c|c|c|}
\hline Case & Rate & $128-256$ & Rate & $256-512$ \\
\hline$l_{2}$ & $2.028 \mathrm{e}+00$ & $1.010 \mathrm{e}-03$ & $1.958 \mathrm{e}+00$ & $2.598 \mathrm{e}-04$ \\
\hline
\end{tabular}

Next, let us consider the convergence of the algorithm in the presence of flow. We take the same initial concentration as in Eq. (48) and the initial velocity is taken to be the rotation

$$
u(x, y)=-\sin ^{2}(\pi x) \sin (2 \pi y), \quad v(x, y)=\sin ^{2}(\pi y) \sin (2 \pi x)
$$

The viscosity $\eta$ is constant and $\mathbf{R e}=100$ and $\mathbf{W e} \mathbf{e}_{s}=100$, which corresponds to the physical Weber number We $=848.5$. No-slip boundary conditions are applied at the boundaries of the domain. The simulation parameters, convergence time and error measurement are as in the case without flow. In tables 7, 8 and 9 the errors and rates of convergence are shown for the concentration $c$ and the velocity components $u$ and $v$, respectively. Observe that these quantities converge with second order accuracy.

Table 7. $l_{2}$-norm of the Errors and Convergence rates for concentration $c$.

\begin{tabular}{||c|c|c|c|c|c||}
\hline Case & $16-32$ & Rate & $32-64$ & Rate & $64-128$ \\
\hline$l_{2}$ & $2.889 \mathrm{e}-01$ & 2.261 & $6.030 \mathrm{e}-02$ & 3.589 & $5.000 \mathrm{e}-03$ \\
\hline
\end{tabular}

\begin{tabular}{||c|c|c|c|c|}
\hline Case & Rate & $128-256$ & Rate & $256-512$ \\
\hline$l_{2}$ & 2.062 & $1.200 \mathrm{e}-03$ & 2.014 & $2.970 \mathrm{e}-04$ \\
\hline
\end{tabular}

Table $8 . l_{2}$-norm of the Errors and Convergence rates for velocity $u$.

\begin{tabular}{||c|c|c|c|c|c|}
\hline Case & $16-32$ & Rate & $32-64$ & Rate & $64-128$ \\
\hline$l_{2}$ & $3.900 \mathrm{e}-03$ & 1.797 & $1.100 \mathrm{e}-03$ & 1.787 & $3.230 \mathrm{e}-04$ \\
\hline
\end{tabular}




\begin{tabular}{||c|c|c|c|c|}
\hline Case & Rate & $128-256$ & Rate & $256-512$ \\
\hline$l_{2}$ & 1.990 & $8.132 \mathrm{e}-05$ & 1.997 & $2.037 \mathrm{e}-05$ \\
\hline
\end{tabular}

Table 9. $l_{2}$-norm of the Errors and Convergence rates for velocity $v$.

\begin{tabular}{||c|c|c|c|c|c|}
\hline Case & $16-32$ & Rate & $32-64$ & Rate & $64-128$ \\
\hline$l_{2}$ & $3.400 \mathrm{e}-03$ & 1.582 & $1.200 \mathrm{e}-03$ & 1.827 & $3.242 \mathrm{e}-04$ \\
\hline
\end{tabular}

\begin{tabular}{||c|c|c|c|c|}
\hline Case & Rate & $128-256$ & Rate & $256-512$ \\
\hline$l_{2}$ & 1.990 & $8.164 \mathrm{e}-05$ & 1.997 & $2.045 \mathrm{e}-05$ \\
\hline
\end{tabular}

In table 10, the errors and convergence rate for the pressure are shown. Here, the rate of convergence appears to be less than second order.

Table 10. $l_{2}$-norm of the Errors and Convergence rates for pressure $p$.

\begin{tabular}{||c|c|c|c|c|c|}
\hline Case & $17-33$ & Rate & $33-65$ & Rate & $65-129$ \\
\hline$l_{2}$ & $8.200 \mathrm{e}-03$ & 1.784 & $2.400 \mathrm{e}-03$ & 2.921 & $3.129 \mathrm{e}-04$ \\
\hline
\end{tabular}

\begin{tabular}{||c|c|c|c|c|}
\hline Case & Rate & $129-257$ & Rate & $257-513$ \\
\hline$l_{2}$ & 1.774 & $9.149 \mathrm{e}-05$ & 1.548 & $3.128 \mathrm{e}-05$ \\
\hline
\end{tabular}

Finally, in table 11, the values of $\delta$ are shown. Observe that $\delta$ converges to zero with second order accuracy.

Table 11. Values of $\delta$ and convergence rate.

\begin{tabular}{||c|c|c|c|c|c|c|}
\hline Case & 16 & Rate & 32 & Rate & 64 & Rate \\
\hline$\delta$ & $-4.827 \mathrm{e}-03$ & 1.5636 & $-1.633 \mathrm{e}-03$ & 1.8942 & $-4.393 \mathrm{e}-04$ & 1.9808 \\
\hline
\end{tabular}




\begin{tabular}{||c|c|c|c|c|c|}
\hline Case & 128 & Rate & 256 & Rate & 512 \\
\hline$\delta$ & $-1.113 \mathrm{e}-04$ & 1.9925 & $-2.797 \mathrm{e}-05$ & 1.9913 & $-7.035 \mathrm{e}-06$ \\
\hline
\end{tabular}

In figure 4, the time evolution of the energy is shown accompanied by the grey-scale contour images of the concentration field at the indicated times. The contour values shown are the same as in the case without flow. At early times, the phases separate and rotate about the center of the domain due to the fluid flow. Between $t=0.7$ and $t=1.0$, the thin necks pinchoff and the lower (upper) bulbs of fluid reconnect with the upper (lower) regions of fluid near the boundaries of the domain and a wiggly interface develops. Due to surface tension, the wiggles straighten out and the bulbs redevelop. At the same time, the rotation slows due to viscous dissipation. The regions of fluid then further straighten, again due to surface tension, to form two horizontal bands. This is the same local equilibrium structure that develops in the case without flow even though the dynamics is very different. However, here the bands consist of fluid originating in both the upper and lower regions of the box while in the case without flow, the fluid in the bands originates from the same region of the box as the band. Observe that the total energy decreases to a constant value consistent with lemma 6 .

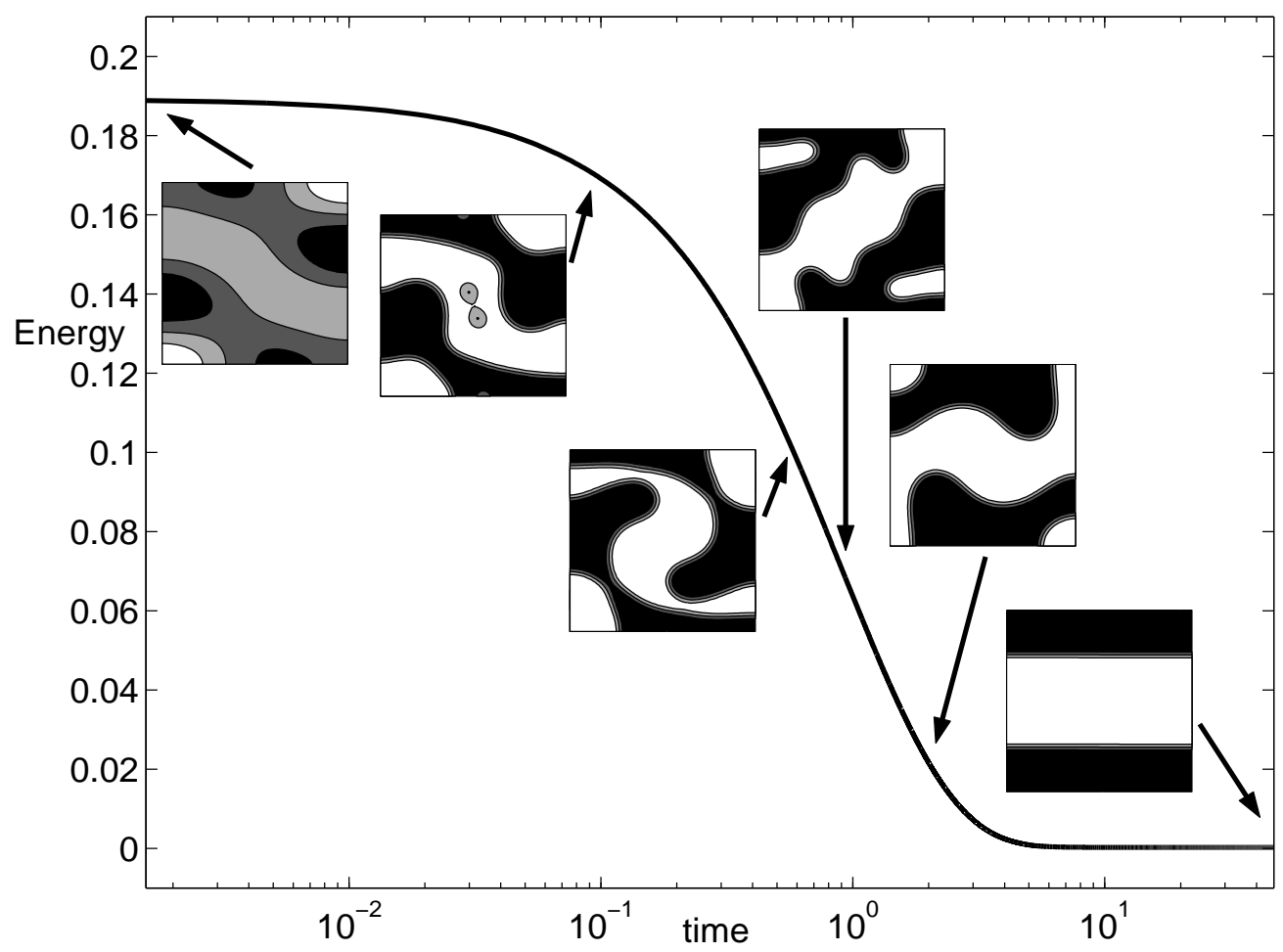

Fig. 4. The time dependent total energy of the numerical solutions with the initial data (48). 


\subsection{Comparison with linear stability theory}

Next, to ensure that we are simulating the correct physical problem, we consider the agreement between the numerics and the results of a linear stability analysis about a constant concentration $c=c_{m}$ and velocity $\mathbf{u}=\mathbf{0}$. Accordingly, we look for a solution of equation (1) of the form

$$
c(x, t)=c_{m}+\sum_{k=1}^{\infty} c^{k}(t) \cos (k \pi x)
$$

where $\left|c^{k}(t)\right| \ll 1$. Neglecting quadratic terms in $c^{k}(t)$, we find that $c^{k}(t)$ must solve the ordinary differential equation,

$$
\frac{d c^{k}}{d t}=-(k \pi)^{2}\left[\phi^{\prime}\left(c_{m}\right)+\epsilon^{2}(k \pi)^{2}\right] c^{k}
$$

Note that there is no flow at the linear level since at that level the pressure balances the extra-stress coupling term in the Navier-Stokes equation. The solution of Eq. (50) is $c^{k}(t)=c^{k}(0) e^{\beta_{k} t}$, where $\beta_{k}=-(k \pi)^{2}\left(\phi^{\prime}\left(c_{m}\right)+\epsilon^{2}(k \pi)^{2}\right)$ is the growth rate. Taking $c_{m}$ in the spinodal region (i.e. $c_{m} \in\left(\frac{3-\sqrt{3}}{6}, \frac{3+\sqrt{3}}{6}\right), \phi^{\prime}\left(c_{m}\right)<0$ ), Eq. (50) shows that the amplitude of a finite number of long wavelength perturbations will grow exponentially in time for sufficiently small $\epsilon$. In particular the fastest growing mode is

$$
k_{\max }=\sqrt{-\phi^{\prime}\left(c_{m}\right) /\left(2 \epsilon^{2} \pi^{2}\right)}
$$

and the growth rate of this mode is $\beta_{k_{\max }}=-\left(k_{\max } \pi\right)^{2} \phi^{\prime}\left(c_{m}\right) / 2$.

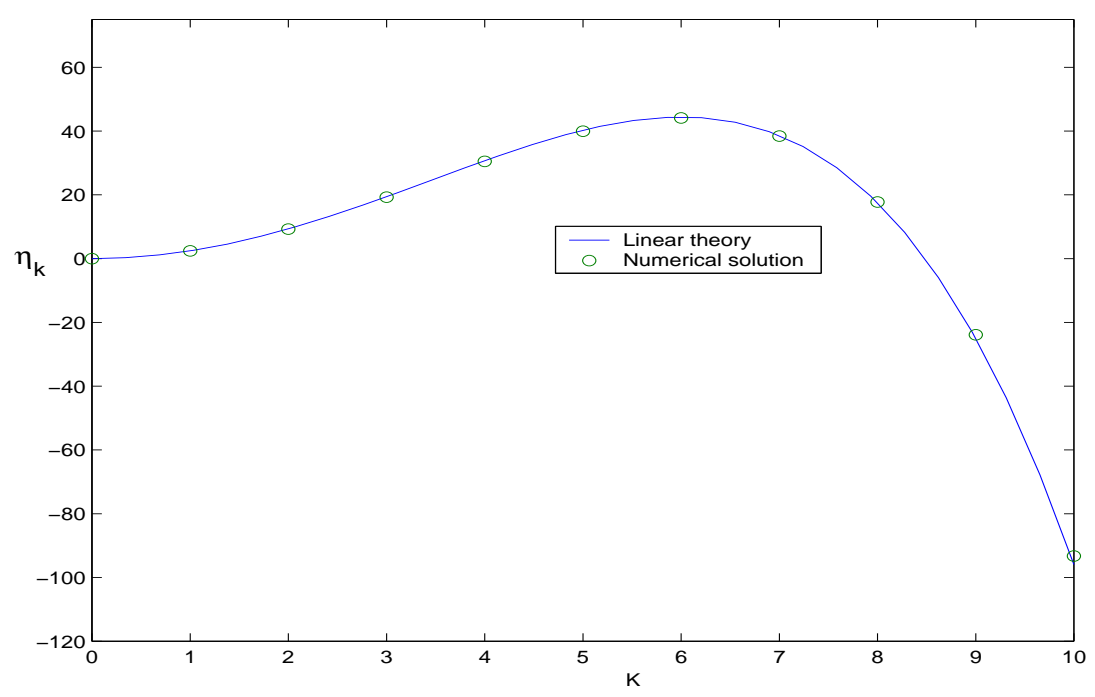

Fig. 5. Growth rate for the different wave numbers k. 
In Fig. 5, the theoretical growth rate $\beta_{k}$ is compared to that obtained from the nonlinear scheme. The numerical growth rate is defined by

$$
\tilde{\beta}_{k}=\log \left(\frac{\max _{i, j}\left|c_{i, j}^{n}-0.5\right|}{\max _{i, j}\left|c_{i, j}^{0}-0.5\right|}\right) / t_{n} .
$$

Here, we used $c_{m}=0.5$, initial data $c_{0}(x)=0.5+0.01 \cos (k \pi x)$ and $\epsilon=0.018757$, $\Delta t=10^{-4}, h=1 / 128$ and $t_{n}=0.01$. The graph shows that the linear analysis (solid line) and numerical solution (circle) are in good agreement.

\subsection{Spinodal decomposition}

In order to demonstrate the effectiveness of our new numerical scheme we consider the effect of flow on spinodal decomposition. We consider an initial concentration field $c^{0}(\mathbf{x})=c_{m}+\xi(\mathbf{x})$, where $c_{m}=0.5$ is the spinodal point and $\xi(\mathbf{x})$ is a random perturbation with magnitude $|\xi(\mathbf{x})| \leq 0.01$. A $64 \times 64$ mesh was used on the square $\Omega=[0,1] \times[0,1]$ with periodic boundary conditions at $x=0$ and 1 . On $y=0$ and 1 we used either no-slip velocity boundary conditions or an imposed shear. Neumann boundary conditions are used for $c$ and $\mu$. Further, we took $\epsilon=0.01, h=1 / 64$, and $\Delta t=0.1 h$. In Fig. 6 the evolution is shown with no flow (left column) and in the presence of surface tension driven flow (right column) with $\mathbf{W e}_{s}=1 /(6 \sqrt{2}$ ) (this corresponds to the physical Weber number $\mathbf{W e}=1$ ). That is, the flow arises only due to surface stresses between the components and we have taken $\mathbf{R e}=1$ and the viscosity $\eta$ is constant. In Fig. 7, the evolution is shown in the absence and presence of surface stress (left and right columns respectively) and with an imposed shear flow, i.e. $(u(x, 1), v(x, 1))=\left(\frac{1}{2}, 0\right)$ and $(u(x, 1), v(x, 1))=\left(-\frac{1}{2}, 0\right)$. In the absence of surface stress, the velocity is the linear field $(u(x, y), v(x, y))=(y-1 / 2,0)$ and satisfies the Navier-Stokes equation (7) with $\mathbf{W e}_{s}=\infty$. In the presence of surface stress, the velocity field is non-linear and we have again used the same non-dimensional constants as given above.

In Figs. 6 and 7, three spatial periods are shown, the $c=0.25,0.5$ and 0.75 contours are shown as filled as before, and the time is constant across a row and increases down the column as indicated in the caption. The times in the corresponding rows for Figs. 6 and 7 are the same. At early times, there is classical spinodal decomposition as the unstable mixture phase separates and regions coalesce. There is little effect of surface stress and flow.

Let us focus first on the effect of surface tension in the absence of shear. In Fig. 6 , at later times, we observe that surface tension acts to decrease the deformation of the interfaces and to reduce their overall length. This causes the fluid fingers to 

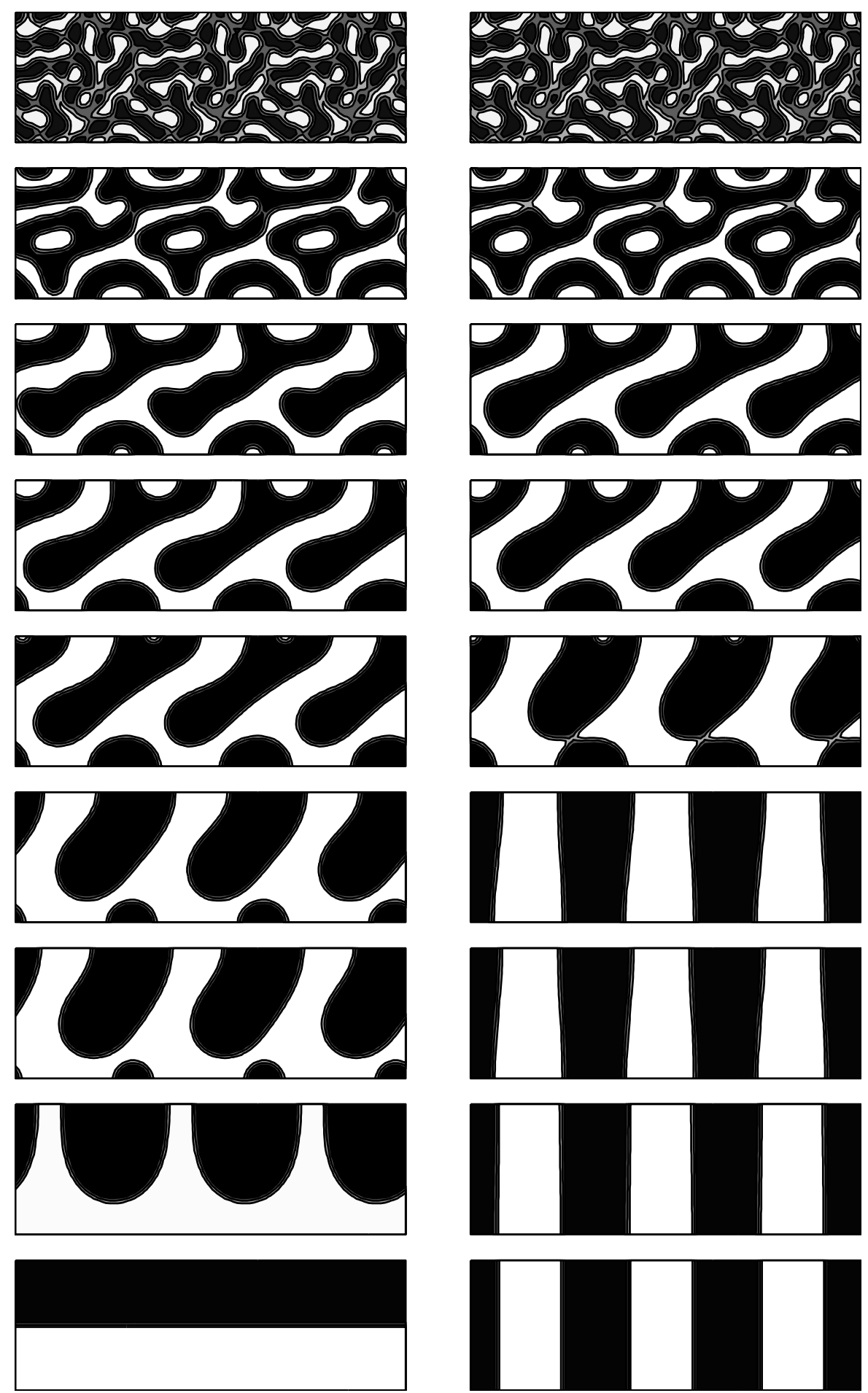

Fig. 6. Evolution with $\mathbf{W e} \mathbf{e}_{s}=\infty$ (left column) and $\mathbf{W e} \mathbf{e}_{s}=1 /(6 \sqrt{2})$ (right column). No applied shear. The times shown are (from top to bottom) $t=0.03,0.16,0.56,0.94,1.88$, $5.63,6.56,17.81$ and 45.94 

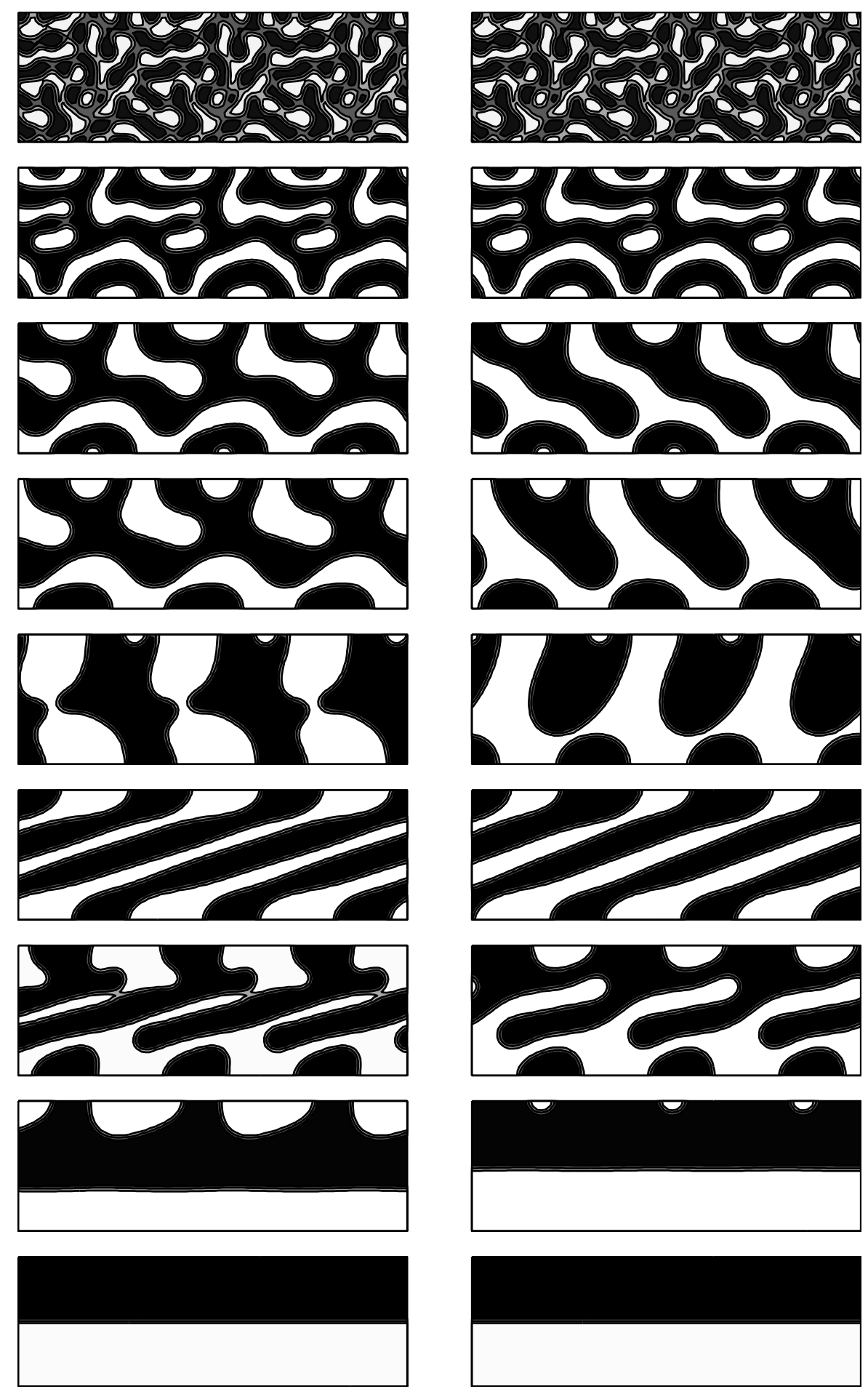

Fig. 7. Evolution with $\mathbf{W e} \mathbf{e}_{s}=\infty$ (left column) and $\mathbf{W e} \mathbf{e}_{s}=1 /(6 \sqrt{2})$ (right column). Applied shear conditions. The times shown are identical to those in figure 6 . 
retract and become more vertical. This leads to the coalescence of the fingers with semi-circular drops at the bottom of the domain. The resulting vertical bands of fluid are a local equilibrium. In the absence of surface tension, the fingers do not coalesce with the drops and classical coarsening occurs as the mass transfers from the drops to the fingers. The fingers then coalesce to form a horizontal band. This is a global energy minimum.

In the presence of shear, we see from Fig. 7, that surface tension has a similar effect and the deformation of interfaces is reduced. Here, however, the morphologies are much more elongated due to the shear. Further, pinchoff and reconnection events occur and the morphology actually repeats itself in time. The stretched bands at $t=5.63$ pinchoff and form drops (see $t=6.56$ ). The drops then reconnect with the fingers (approximately at $t=7.0$, not shown) and re-form the stretched bands. When $\mathbf{W e}_{s}=\infty$, this sequence occurs twice while when $\mathbf{W e} \mathbf{e}_{s}=1 /(6 \sqrt{2})$ this sequence occurs three times since the fingers retract more due to surface tension which enhances their capability to coalesce. This temporal periodicity is discussed further below. Eventually, the periodicity is broken since the drops that are formed become smaller with each cycle. After the last cycle, there is no reconnection. The fingers then merge to form a single horizontal band which is the global energy minimum.

In Fig. 8 the total energy (27) evolution for the different simulations is plotted versus time. In the absence of shear (dot-dashed: $\mathbf{W e}_{s}=1 /(6 \sqrt{2})$ and dashed: $\mathbf{W e} \mathbf{e}_{s}=$ $\infty)$, the energy decreases monotonically as predicted by lemma 6 . In the presence of shear (large dots: $\mathbf{W e}_{s}=1 /(6 \sqrt{2})$ and small dots: $\mathbf{W e} \mathbf{e}_{s}=\infty$ ), there are energy oscillations. Observe that there are three oscillations when $\mathbf{W e}_{s}=1 /(6 \sqrt{2})$ and two when $\mathbf{W e}_{s}=\infty$. These correspond to the pinchoff and reconnection scenarios described above.

In Fig. 9, we present the scaled kinetic (dashed) and surface energy (solid) evolutions through first oscillation ending approximately at $t=9.0$. In addition, we show the concentration morphology at the indicated times. To obtain the scaled kinetic energy, the actual kinetic energy is multiplied by 100. At early times, energy is transferred from the surface to the fluid while at later times this process is reversed as the interfaces stretch and elongate. The peaks seen in the kinetic energy correspond to topology transitions of the concentration field in the flow. For example, lower ends of the stretched bands pinchoff to form drops at approximately $t=6.4$. The drops then recoalesce with the fingers at approximately $t=7.0$. Each of these events is seen to be associated with a local peak in the kinetic energy. Energy is transmitted to the fluid through the large surface stress that develops during the transition and subsequent retraction of the fingers.

To understand further the oscillation of the energy in the presence of shear, we 
compare in Fig. 10 the difference quotient

$$
\frac{\mathcal{E}_{h}\left(c^{n+1}, \mathbf{u}^{n+1}\right)-\mathcal{E}_{h}\left(c^{n}, \mathbf{u}^{n}\right)}{\Delta t}
$$

shown as circles to the corresponding spatial discretization of the analytical value $\frac{d}{d t} \mathcal{E}\left(c^{n+\frac{1}{2}}, \mathbf{u}^{n+\frac{1}{2}}\right)$ given by

$$
\frac{1}{\mathbf{R e}}\left(\mathbf{u}^{n+\frac{1}{2}}, \Delta \mathbf{u}^{n+\frac{1}{2}}\right)_{h}-\frac{\mathbf{W e}_{s}^{-1}}{\epsilon}\left(\left|\nabla \mu^{n+\frac{1}{2}}\right|^{2}, 1\right)_{h}-\frac{\mathbf{W e}_{s}^{-1}}{\epsilon \Delta t}\left(\left(c^{n+1}-c^{n}\right)^{4}, 1\right)_{h}
$$

shown as the solid line. Observe that there is excellent agreement between these two independently calculated quantities. This shows that the oscillations are due to the flow through the shear boundary condition and provides an independent validation of the accuracy of our numerical results.

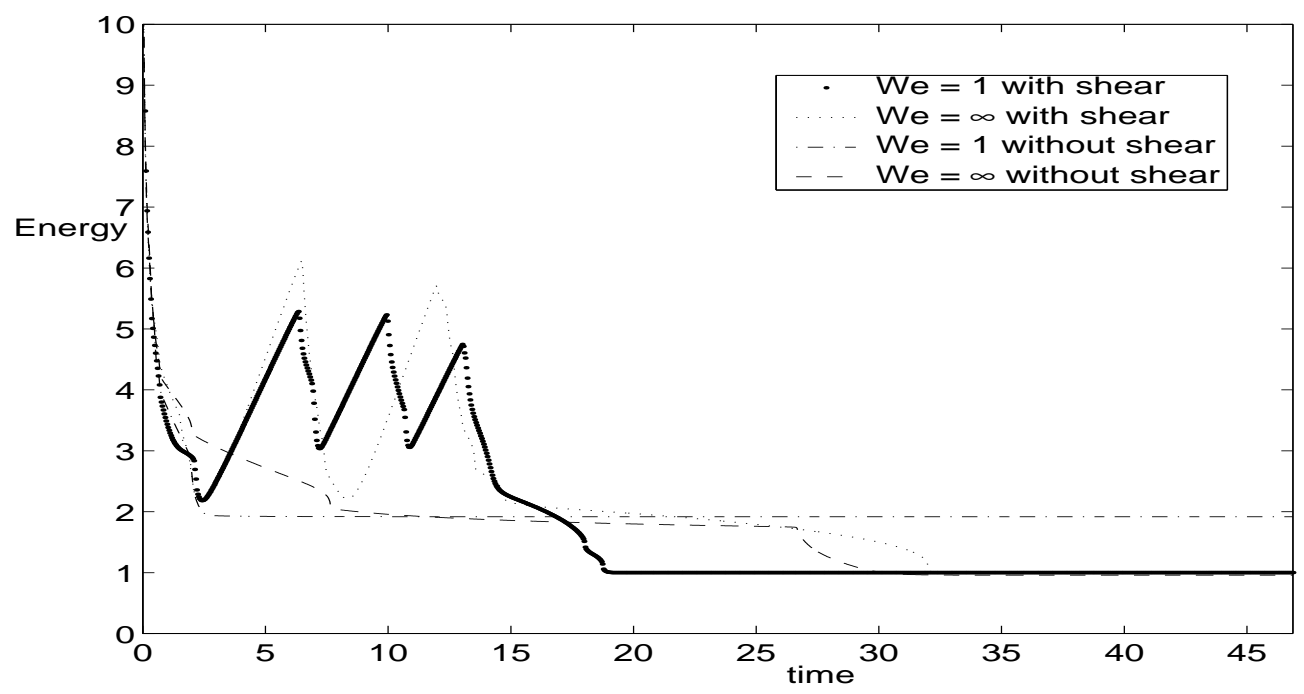

Fig. 8. Energy evolution under the flow different conditions.

\section{Conclusions}

In this paper, we have developed a conservative, $2^{\text {nd }}$ order accurate fully implicit discretization of the NSCH system that has an associated discrete energy functional. In addition, the scheme has a straightforward extension to multi-component systems. This will be exploited in a future work where we examine fluid flows with three constituent components [38].

To efficiently solve the discrete system at the implicit time-level, we have developed a nonlinear multigrid method to solve the $\mathbf{C H}$ equation which is then coupled to a projection method that is used to solve the NS equation. We have analyzed and proved convergence of the scheme in the absence of flow. The convergence in 


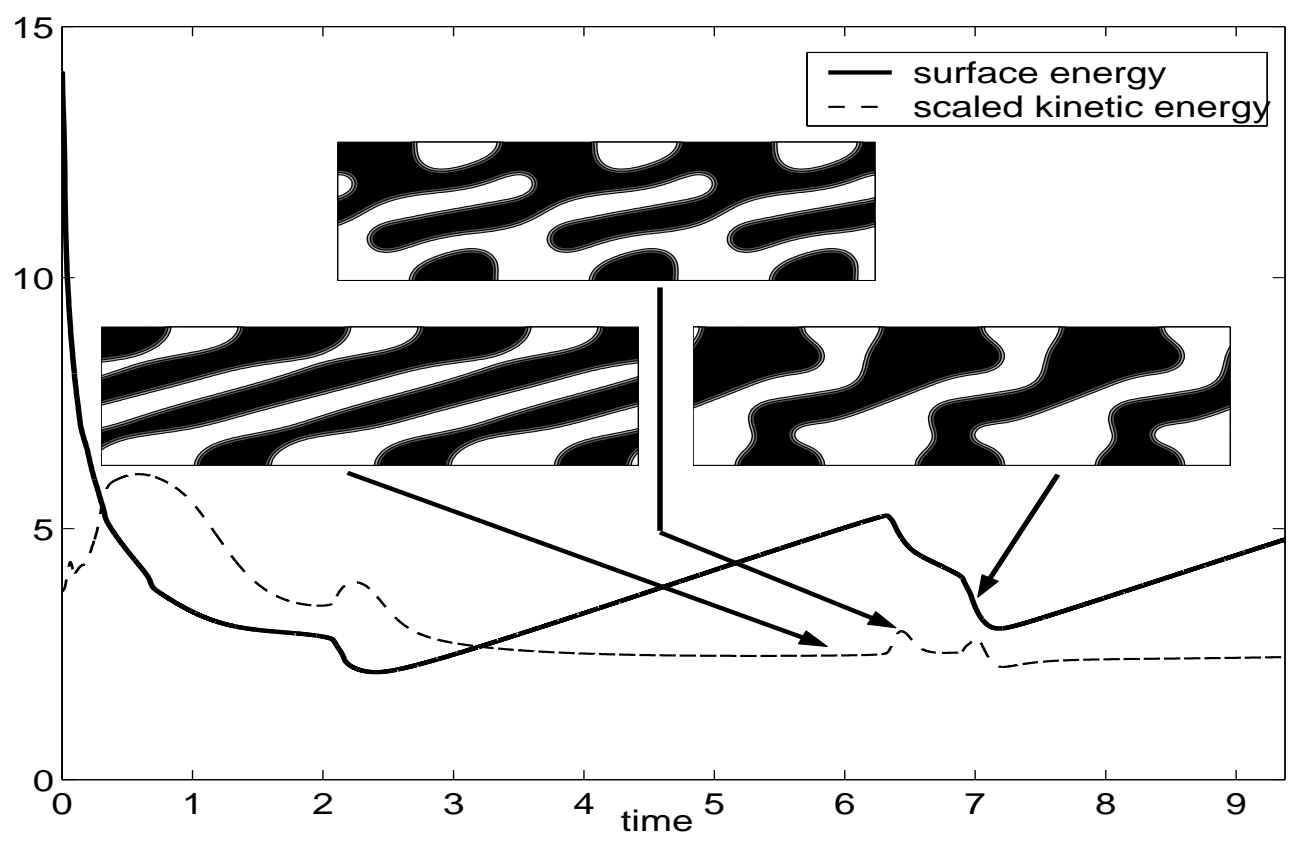

Fig. 9. Surface energy and scaled kinetic energy.

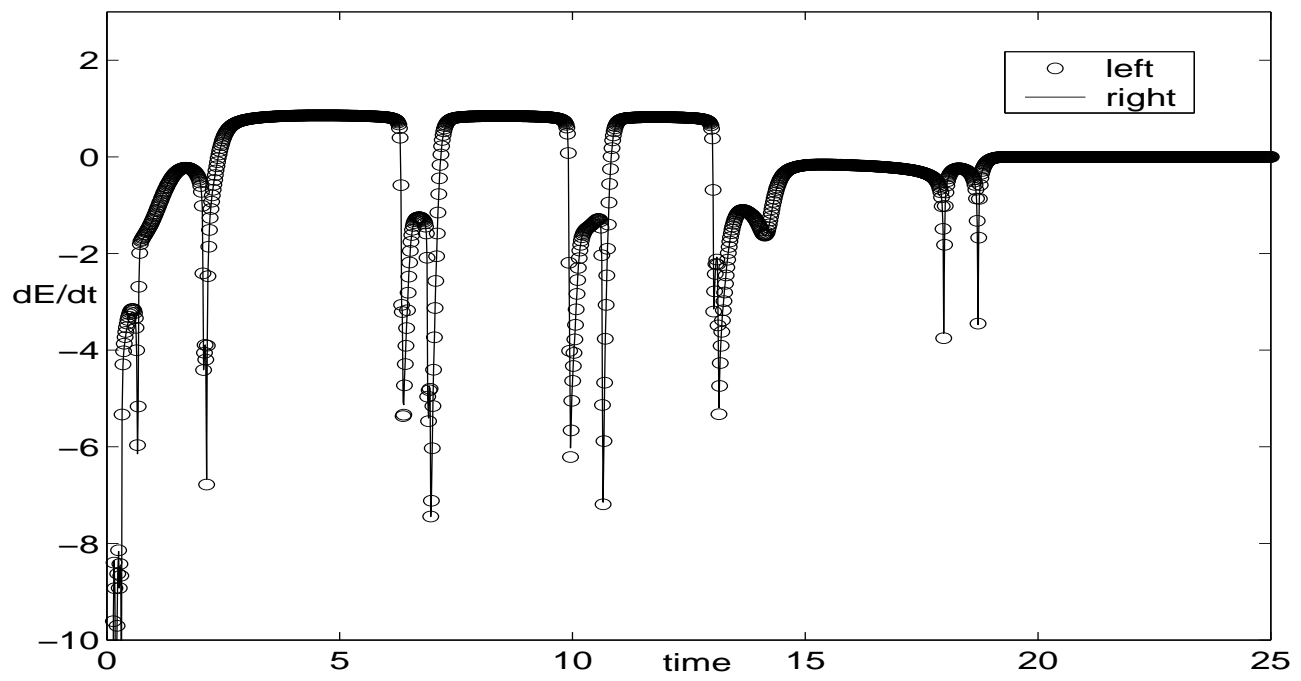

Fig. 10. Comparison of Eqs. (51), circles, and (52), solid line.

the presence of flow is currently under study. We demonstrated convergence of our scheme numerically in both the presence and absence of flow and performed simulations of phase separation via spinodal decomposition. We examined the separate effects of surface tension and external flow on the decomposition. We found surface tension driven flow alone increases coalescence rates through the retraction of interfaces. When there is an external shear flow, the evolution of the flow is nontrivial and the flow morphology repeats itself in time as multiple pinchoff and reconnection events occur. Eventually, the periodic motion ceases and the system relaxes to a global equilibrium. The equilibria we observe appears has a similar structure in all cases although the dynamics of the evolution is quite different. 
We view the work presented in this paper as preparatory for the detailed investigation of liquid/liquid interfaces with surface tension where the interfaces separate two immiscible fluids. One such example is the break-up of an axisymmetric thread of viscous fluid surrounded by another liquid due to the Rayleigh instability. In figure 11, the break-up of such a thread is shown using an axisymmetric version of the NSCH algorithm presented in this paper. In this simulation, the initial condition consists of a column of fluid with radius 0.5 perturbed by a cosine perturbation with an amplitude of 0.05 . The initial velocity is equal to zero. The inner and outer fluids are have the same density and viscosity. The Reynolds number (using the characteristic velocity scale that is proportional to the ratio of surface tension and viscosity and length scale that is proportional to the radius of the column) is $\mathbf{R e}=6.0$. The physical Weber number is $\mathbf{W e}=0.18$, which corresponds to $\mathbf{W e}_{s}=0.021$. The time step $\Delta t=1 \times 10^{-3}$, the domain is $0 \leq r \leq \pi / 2$ and $0 \leq z \leq 2 \pi$. The grid size is $h=\pi / 128 \approx 0.049$ in both $r$ and $z$. Periodic boundary conditions are imposed at $z=0$ and $2 \pi$. Neumann and no-slip boundary conditions are imposed at $r=\pi / 2$ for $c, \mu$ and $\mathbf{u}$ respectively. Finally, $\epsilon=0.02$ and $\mathbf{P e}=1 / \epsilon$. In the simulation presented in figure 11, a neck is seen to develop and pinch off to form three droplets at approximately time $t=2.5$. At this first pinchoff, there is a slight overturning of the interface in the neck region (see $t=1.8$ ). At later times, the main drops circularize due to surface tension while the smaller daughter drops undergo additional pinchoff events. These secondary pinchoff events appear to be due to the Rayleigh instability acting upon the small drops as the small drops oscillate. In each event, it is the outermost drops that pinchoff. This significantly reduces the size of the drops and eventually leads to steady state in which a distribution of small drops separates the larger ones. The small drops are nearly monodisperse in size. This result will be investigated in more detail in [37].

\section{A Ternary Cahn-Hilliard system}

Let us show how to extend the scheme (13)-(15) to the case of three species (ternary). This system can be derived from the energy functional

$$
\mathbf{J}\left(c_{1}, c_{2}\right)=\int_{\Omega}\left[F\left(c_{1}, c_{2}\right)+\frac{\epsilon_{1}^{2}}{2}\left|\nabla c_{1}\right|^{2}+\frac{\epsilon_{2}^{2}}{2}\left|\nabla c_{2}\right|^{2}+\frac{\epsilon_{3}^{2}}{2}\left|\nabla\left(1-c_{1}-c_{2}\right)\right|^{2}\right] d \mathbf{x} .
$$

For simplicity, we consider a typical free energy given by fourth order polynomial

$$
F\left(c_{1}, c_{2}\right)=c_{1}^{2}{c_{2}}^{2}+c_{2}{ }^{2} c_{3}^{2}+c_{3}^{2} c_{1}^{2}=c_{1}^{2} c_{2}^{2}+\left(c_{1}^{2}+c_{2}{ }^{2}\right)\left(1-c_{1}-c_{2}\right)^{2},
$$

where $c_{1}, c_{2}$, and $c_{3}$ as concentrations of three different species and $c_{1}+c_{2}+c_{3}=1$. From this constraint, we have the following system, 


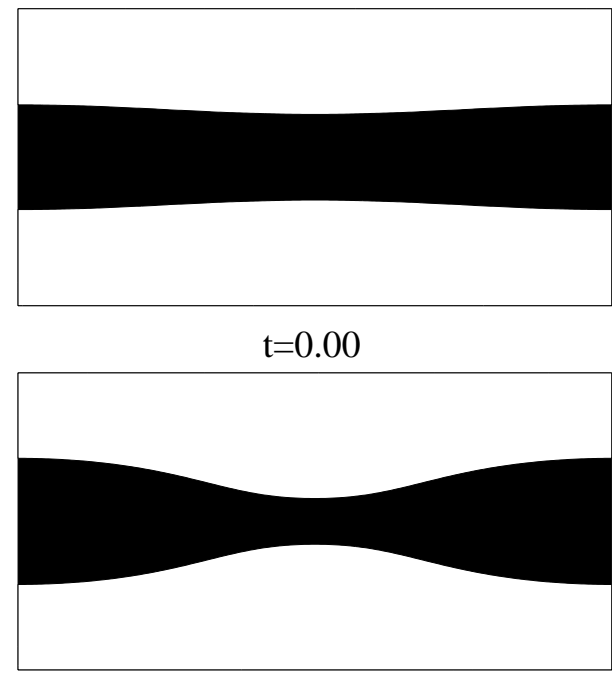

$\mathrm{t}=1.4$

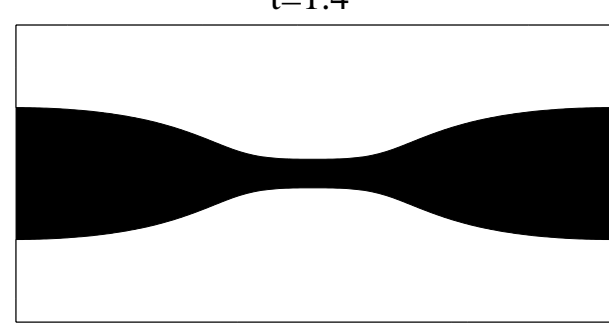

$\mathrm{t}=1.6$

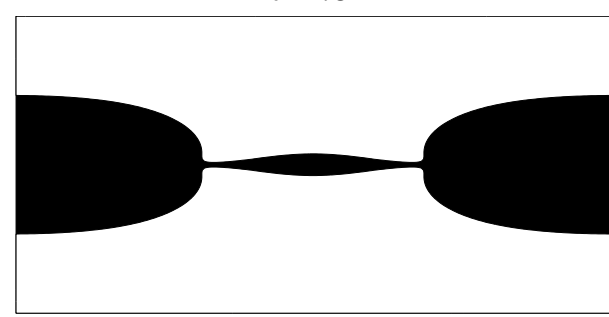

$\mathrm{t}=1.8$

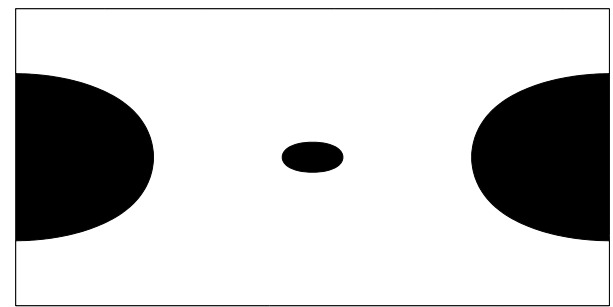

$\mathrm{t}=2.8$

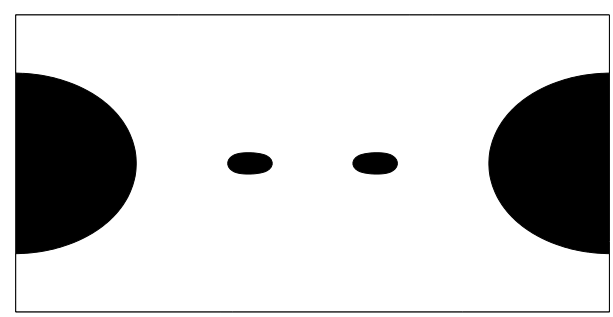

$\mathrm{t}=5.8$

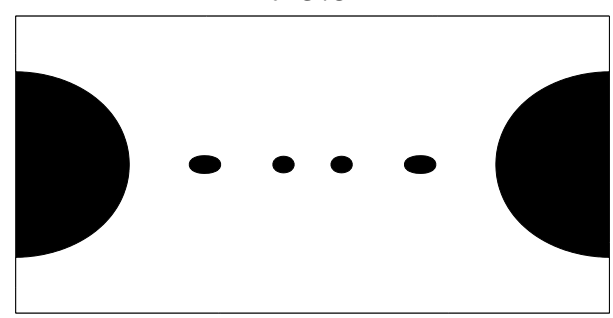

$\mathrm{t}=7.8$

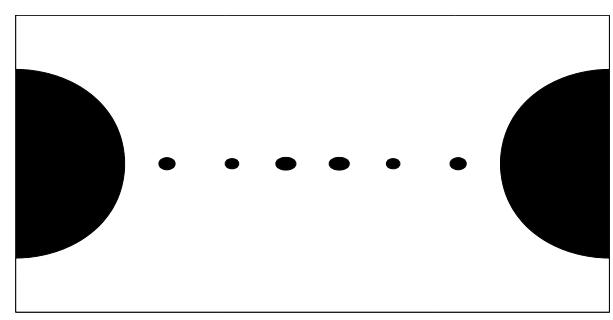

$\mathrm{t}=10.0$

Fig. 11. Time evolution of a highly extended fluid suspended in another fluid. The dimensionless times are shown below each figure.

$$
\begin{array}{lll}
\frac{\partial c_{1}}{\partial t}=\Delta \mu_{1}, & \mu_{1}=\frac{F\left(c_{1}, c_{2}\right)}{\partial c_{1}}-\left(\epsilon_{1}^{2}+\epsilon_{3}^{2}\right) \Delta c_{1}-\epsilon_{3}^{2} \Delta c_{2}, & \text { in } \Omega \times(0, T), \\
\frac{\partial c_{2}}{\partial t}=\Delta \mu_{2}, & \mu_{2}=\frac{F\left(c_{1}, c_{2}\right)}{\partial c_{2}}-\left(\epsilon_{2}^{2}+\epsilon_{3}^{2}\right) \Delta c_{2}-\epsilon_{3}^{2} \Delta c_{1}, & \text { in } \Omega \times(0, T),
\end{array}
$$

subject to initial conditions

$$
c_{1}(\mathbf{x}, 0)=c_{10}(\mathbf{x}), \quad c_{2}(\mathbf{x}, 0)=c_{20}(\mathbf{x}) \quad \mathbf{x} \in \Omega
$$

with homogeneous Neumann boundary conditions. The discretization of this ternary system is a straightforward extension of the binary scheme (13) and (14): 


$$
\begin{aligned}
& \frac{c_{1 i j}^{n+1}}{\Delta t}-\Delta_{d} \mu_{1 i j}^{n+\frac{1}{2}}=\frac{c_{1 i j}^{n}}{\Delta t}, \\
& \frac{c_{2 i j}^{n+1}}{\Delta t}-\Delta_{d} \mu_{2 i j}^{n+\frac{1}{2}}=\frac{c_{2 i j}^{n}}{\Delta t},
\end{aligned}
$$

where

$$
\begin{aligned}
\mu_{1 i j}^{n+\frac{1}{2}}-\hat{\phi}\left(c_{1 i j}^{n}, c_{1 i j}^{n+1}, c_{2 i j}^{n}, c_{2 i j}^{n+1}\right)_{1} & +\frac{\epsilon_{1}^{2}+\epsilon_{3}^{2}}{2} \Delta_{d} c_{1 i j}^{n+1}+\frac{\epsilon_{3}^{2}}{2} \Delta_{d} c_{2 i j}^{n+1} \\
& =-\frac{\epsilon_{1}^{2}+\epsilon_{3}^{2}}{2} \Delta_{d} c_{1 i j}^{n}-\frac{\epsilon_{3}^{2}}{2} \Delta_{d} c_{2 i j}^{n}, \\
\mu_{2 i j}^{n+\frac{1}{2}}-\hat{\phi}\left(c_{1 i j}^{n}, c_{1 i j}^{n+1}, c_{2 i j}^{n}, c_{2 i j}^{n+1}\right)_{2} & +\frac{\epsilon_{2}^{2}+\epsilon_{3}^{2}}{2} \Delta_{d} c_{2 i j}^{n+1}+\frac{\epsilon_{3}^{2}}{2} \Delta_{d} c_{1 i j}^{n+1} \\
& =-\frac{\epsilon_{2}^{2}+\epsilon_{3}^{2}}{2} \Delta_{d} c_{2 i j}^{n}-\frac{\epsilon_{3}^{2}}{2} \Delta_{d} c_{1 i j}^{n},
\end{aligned}
$$

and

$$
\begin{aligned}
\hat{\phi}\left(c_{1}{ }^{n}, c_{1}{ }^{n+1}, c_{2}{ }^{n}, c_{2}{ }^{n+1}\right)_{1}= & \partial_{c_{1}} F\left(c_{1}{ }^{n+1}, c_{2}{ }^{n+1}\right) \\
& +\frac{1}{2} \partial_{c_{1} c_{1}} F\left(c_{1}{ }^{n+1}, c_{2}{ }^{n+1}\right)\left(c_{1}{ }^{n}-c_{1}{ }^{n+1}\right) \\
& +\cdots+\frac{1}{4 !} \partial_{c_{2} c_{2} c_{2} c_{1}} F\left(c_{1}{ }^{n+1}, c_{2}{ }^{n+1}\right)\left(c_{2}{ }^{n}-c_{2}{ }^{n+1}\right)^{3}, \\
\hat{\phi}\left(c_{1}{ }^{n}, c_{1}{ }^{n+1}, c_{2}{ }^{n}, c_{2}{ }^{n+1}\right)_{2}= & \partial_{c_{2}} F\left(c_{1}^{n+1}, c_{2}{ }^{n+1}\right) \\
& +\frac{1}{2} \partial_{c_{1} c_{2}} F\left(c_{1}{ }^{n+1}, c_{2}{ }^{n+1}\right)\left(c_{1}{ }^{n}-c_{1}{ }^{n+1}\right) \\
& +\cdots+\frac{1}{4 !} \partial_{c_{2} c_{2} c_{2} c_{2}} F\left({c_{1}}^{n+1}, c_{2}{ }^{n+1}\right)\left(c_{2}{ }^{n}-c_{2}{ }^{n+1}\right)^{3},
\end{aligned}
$$

where $\hat{\phi}(\ldots)_{1}$ and $\hat{\phi}(\ldots)_{2}$ denote Taylor series approximations to $\partial_{c_{1}} F$ and $\partial_{c_{2}} F$ respectively. This scheme has a discrete energy functional given by a discretization of (A.1) analogous to Eq. (25) in the binary case.

Next, the nondimensional, ternary incompressible generalized NS equation is:

$$
\begin{aligned}
\nabla \cdot \mathbf{u}= & 0 \\
\mathbf{u}_{t}+\omega \times \mathbf{u}= & -\nabla P+\frac{1}{\mathbf{R e}} \nabla \cdot\left[\eta\left(c_{1}, c_{2}\right)\left(\nabla \mathbf{u}+\nabla \mathbf{u}^{T}\right)\right] \\
& -\frac{\mathbf{W e}_{s}^{-1}}{2 \epsilon_{i}} c_{i} \nabla \mu_{i} .
\end{aligned}
$$


Taking a discretization of this system analogous to that in the binary case (e.g. Eqs. (18) - (20)), and adding advection terms to Eqs. (A.2) and (A.3), it is straightforward to show that this scheme has a discrete energy functional analogous to Eq. (27).

\section{B Smoothing operator}

The derivation of the $S M O O T H$ relaxation operator given in Eqs. (34) and (35) is as follows. Rewriting (45), we get

$$
\frac{c_{i j}^{n+1}}{\Delta t}+\left(\frac{2}{\Delta x^{2}}+\frac{2}{\Delta y^{2}}\right) \mu_{i j}^{n+\frac{1}{2}}=\left(\frac{\mu_{i+1, j}^{n+\frac{1}{2}}+\mu_{i-1, j}^{n+\frac{1}{2}}}{\Delta x^{2}}+\frac{\mu_{i, j+1}^{n+\frac{1}{2}}+\mu_{i, j-1}^{n+\frac{1}{2}}}{\Delta y^{2}}\right)+\frac{c_{i j}^{n}}{\Delta t} .
$$

By Eq. (46),

$$
\begin{aligned}
-\frac{\epsilon^{2}}{2}\left(\frac{2}{\Delta x^{2}}+\right. & \left.\frac{2}{\Delta y^{2}}\right) c_{i j}^{n+1}+\mu_{i j}^{n+\frac{1}{2}}=\frac{1}{2} \epsilon^{2} \Delta_{d} c_{i j}^{n}+\hat{\phi}\left(c_{i j}^{n}, c_{i j}^{n+1}\right) \\
& -\frac{\epsilon^{2}}{2 \Delta x^{2}}\left(c_{i+1, j}^{n+1}+c_{i-1, j}^{n+1}\right)-\frac{\epsilon^{2}}{2 \Delta y^{2}}\left(c_{i, j+1}^{n+1}+c_{i, j-1}^{n+1}\right) .
\end{aligned}
$$

Since $\hat{\phi}\left(c_{i j}^{n}, c_{i j}^{n+1}\right)$ is nonlinear with respect to $c_{i j}^{n+1}$, we linearize $\hat{\phi}\left(c_{i j}^{n}, c_{i j}^{n+1}\right)$ at $c_{i j}^{m}$

$$
\hat{\phi}\left(c_{i j}^{n}, c_{i j}^{n+1}\right) \approx \hat{\phi}\left(c_{i j}^{n}, c_{i j}^{m}\right)+\frac{\partial \hat{\phi}\left(c_{i j}^{n}, c_{i j}^{m}\right)}{\partial c_{2}}\left(c_{i j}^{n+1}-c_{i j}^{m}\right) .
$$

After substitution of this into (B.1), we get

$$
\begin{aligned}
-\left[\frac{\epsilon^{2}}{2}\left(\frac{2}{\Delta x^{2}}+\frac{2}{\Delta y^{2}}\right)+\right. & \left.\frac{\partial \hat{\phi}\left(c_{i j}^{n}, c_{i j}^{m}\right)}{\partial c_{2}}\right] c_{i j}^{n+1}+\mu_{i j}^{n+\frac{1}{2}} \\
= & \frac{\epsilon^{2}}{2} \Delta c_{i j}^{n}+\hat{\phi}\left(c_{i j}^{n}, c_{i j}^{m}\right)-\frac{\partial \hat{\phi}\left(c_{i j}^{n}, c_{i j}^{m}\right)}{\partial c_{2}} c_{i j}^{m} \\
& -\frac{\epsilon^{2}}{2 \Delta x^{2}}\left(c_{i+1, j}^{n+1}+c_{i-1, j}^{n+1}\right)-\frac{\epsilon^{2}}{2 \Delta y^{2}}\left(c_{i, j+1}^{n+1}+c_{i, j-1}^{n+1}\right) .
\end{aligned}
$$

Next, replace $c_{i-1, j}^{n+1}, c_{i, j}^{n+1}$ and $c_{i, j-1}^{n+1}$ with $\bar{c}_{i-1, j}^{m}, \bar{c}_{i, j}^{m}$ and $\bar{c}_{i, j-1}^{m}$ respectively. The other two terms $c_{i+1, j}^{n+1}$ and $c_{i, j+1}^{n+1}$ are replaced with $c_{i+1, j}^{m}$ and $c_{i, j+1}^{m}$ respectively. The chemical potential is replaced analogously with the time index being set to $m-\frac{1}{2}$. We thus obtain the relaxation scheme:

$$
\frac{\bar{c}_{i j}^{m}}{\Delta t}+\left(\frac{2}{\Delta x^{2}}+\frac{2}{\Delta y^{2}}\right) \bar{\mu}_{i j}^{m-\frac{1}{2}}=\frac{\mu_{i+1, j}^{m-\frac{1}{2}}+\bar{\mu}_{i-1, j}^{m-\frac{1}{2}}}{\Delta x^{2}}+\frac{\mu_{i, j+1}^{m-\frac{1}{2}}+\bar{\mu}_{i, j-1}^{m-\frac{1}{2}}}{\Delta y^{2}}+\frac{c_{i j}^{n}}{\Delta t},
$$


and

$$
\begin{aligned}
-\left[\frac{\epsilon^{2}}{2}\left(\frac{2}{\Delta x^{2}}+\frac{2}{\Delta y^{2}}\right)+\right. & \left.\frac{\partial \hat{\phi}\left(c_{i j}^{n}, c_{i j}^{m}\right)}{\partial c_{2}}\right] \bar{c}_{i j}^{m}+\bar{\mu}_{i j}^{m-\frac{1}{2}} \\
= & -\frac{\epsilon^{2}}{2} \Delta c_{i j}^{n}+\hat{\phi}\left(c_{i j}^{n}, c_{i j}^{m}\right)-\frac{\partial \hat{\phi}\left(c_{i j}^{n}, c_{i j}^{m}\right)}{\partial c_{2}} c_{i j}^{m} \\
& -\frac{\epsilon^{2}}{2 \Delta x^{2}}\left(c_{i+1, j}^{m}+\bar{c}_{i-1, j}^{m}\right)-\frac{\epsilon^{2}}{2 \Delta y^{2}}\left(c_{i, j+1}^{m}+\bar{c}_{i, j-1}^{m}\right),
\end{aligned}
$$

as claimed in section 3 .

\section{Multigrid method for the intermediate velocity field $\mathbf{u}^{*}$}

For simplicity, let $\mathbf{u}=\mathbf{u}^{*, k+1}$, then we have

$$
L \mathbf{u}=(f, g)
$$

where

$$
L=\frac{1}{\Delta t}-\frac{1}{2 \mathbf{R e}} \Delta_{d}
$$

and

$$
\begin{array}{r}
(f, g)=-\frac{1}{2} \omega^{n+\frac{1}{2}, k} \times\left(\mathbf{u}^{n+1, k}\right. \\
\left.+\mathbf{u}^{n}\right)-\tilde{\nabla}_{d}^{c} P^{n+\frac{1}{2}, k}+\frac{1}{2 \mathbf{R e}} \Delta_{d} \mathbf{u}^{n} \\
-\frac{1}{2 \epsilon \mathbf{W e}}\left(c^{n+1, k}+c^{n}\right) \nabla_{d} \mu^{n+\frac{1}{2}, k}
\end{array}
$$

Since operator $L$ is a linear operator, we use a linear multigrid method to solve Eq. (C.1).

\section{Multigrid cycle}

$$
\mathbf{u}_{k}^{m+1}=M G C Y C\left(k, \mathbf{u}_{k}^{m}, L_{k}, f_{k}^{n}, g_{k}^{n}, \nu\right)
$$

Now, define the MGCYC.

\section{(1) Presmoothing}


Compute $\overline{\mathbf{u}}_{k}^{m}$ by applying $\nu$ smoothing steps to $\mathbf{u}_{k}^{m}$

$$
\overline{\mathbf{u}}_{k}^{m}=\operatorname{SMOOTH}^{\nu}\left(\mathbf{u}_{k}^{m}, L_{k}, f_{k}^{n}, g_{k}^{n}\right),
$$

One $S M O O T H$ operator step consists of relaxing the system (C.1) given below for each $i$ and $j$.

$$
\begin{aligned}
& \bar{u}_{i j}^{m}=\left(f+\frac{u_{i+1, j}^{m}+\bar{u}_{i-1, j}^{m}+u_{i, j+1}^{m}+\bar{u}_{i, j-1}^{m}}{2 h^{2} \mathbf{R e}}\right) /\left(\frac{1}{\Delta t}+\frac{2}{h^{2} \mathbf{R e}}\right), \\
& \bar{v}_{i j}^{m}=\left(g+\frac{v_{i+1, j}^{m}+\bar{v}_{i-1, j}^{m}+v_{i, j+1}^{m}+\bar{v}_{i, j-1}^{m}}{2 h^{2} \mathbf{R e}}\right) /\left(\frac{1}{\Delta t}+\frac{2}{h^{2} \mathbf{R e}}\right) .
\end{aligned}
$$

\section{(2) Compute the defect}

$$
\left(\bar{d}_{1 k}^{m}, \bar{d}_{2 k}^{m}\right)=\left(f_{k}^{n}, g_{k}^{n}\right)-L_{k}\left(\bar{u}_{k}^{m}, \bar{v}_{k}^{m}\right)
$$

\section{(3) Restrict the defect}

$$
\left(\bar{d}_{1 k-1}^{m}, \bar{d}_{2 k-1}^{m}\right)=I_{k}^{k-1}\left(\bar{d}_{1 k}^{m}, \bar{d}_{2 k}^{m}\right)
$$

(4) Compute an approximate solution $\left\{\hat{u}_{k-1}^{m}, \hat{v}_{k-1}^{m}\right\}$ of the coarse grid equation on $\Omega_{k-1}$, i.e.

$$
L_{k-1}\left(u_{k-1}^{m}, v_{k-1}^{m}\right)=\left(\bar{d}_{1 k-1}^{m}, \bar{d}_{2 k-1}^{m}\right) .
$$

If $k=1$, we use a direct solver. If $k>1$, we solve (C.2) by performing a $k$-grid cycle using the zero grid function as an initial approximation:

$$
\hat{\mathbf{v}}_{k-1}^{m}=\operatorname{MGCYC}\left(k-1, \mathbf{0}, L_{k-1}, \bar{d}_{1 k-1}^{m}, \bar{d}_{2 k-1}^{m}, \nu\right) .
$$

\section{(5) Interpolate the correction}

$$
\begin{aligned}
& \hat{v}_{1 k}^{m}=I_{k-1}^{k} \hat{v}_{1 k-1}^{m} . \\
& \hat{v}_{2 k}^{m}=I_{k-1}^{k} \hat{v}_{2 k-1}^{m} .
\end{aligned}
$$

(6) Compute the corrected approximation on $\Omega_{k}$

$$
\begin{aligned}
& u_{k}^{m, \text { after } C G C}=\bar{u}_{k}^{m}+\hat{v}_{1 k}^{m} . \\
& v_{k}^{m, \text { after } C G C}=\bar{v}_{k}^{m}+\hat{v}_{2 k}^{m} .
\end{aligned}
$$

\section{(7) Postsmoothing}


Compute $\mathbf{u}_{k}^{m+1}$ by applying $\nu$ smoothing steps to $\mathbf{u}_{k}^{m}$, after $C G C$,

$$
\mathbf{u}_{k}^{m+1}=\operatorname{SMOOTH} H^{\nu}\left(\mathbf{u}_{k}^{m} \text {, after } C G C, L_{k}, f_{k}^{n}, g_{k}^{n}\right) .
$$

This completes the description of the multigrid cycle.

\section{Crank-Nicholson scheme}

Here, we present another Crank-Nicholson scheme in which

$$
\hat{\phi}\left(c^{n}, c^{n+1}\right)=\frac{1}{2}\left(\phi\left(c^{n}\right)+\phi\left(c^{n+1}\right)\right) .
$$

This results in the more traditional scheme:

$$
\begin{aligned}
& \frac{c_{i j}^{n+1}-c_{i j}^{n}}{\Delta t}=\Delta_{d} \mu_{i j}^{n+\frac{1}{2}}, \\
& \mu_{i j}^{n+1 / 2}=\frac{1}{2}\left(\phi\left(c_{i j}^{n+1}\right)+\phi\left(c_{i j}^{n}\right)\right)-\frac{\epsilon^{2}}{2} \Delta_{d}\left(c_{i j}^{n+1}+c_{i j}^{n}\right) .
\end{aligned}
$$

This scheme is second order accurate and convergence may be proved using an argument analogous to that given in theorem 9. Further, this algorithm extends straightforwardly to the ternary case and the nonlinear multigrid method given in section 3 also can easily be modified to solve the corresponding nonlinear system at the implicit time level. Moreover, at the linear level (i.e. $\phi$ is a linear function), this scheme is the same as that considered in the body of the paper. However, at the nonlinear level, we are unable to prove that the system given above has a discrete energy function unless a second order time step constraint is imposed. This constraint is much stronger than that needed for stability and seems to be a shortcoming of the analysis as simulation results always yield non-increasing discrete energies.

\section{Acknowledgments}

The first (J.S. Kim) and third (J. S. Lowengrub) authors acknowledge the support of the Department of Energy, Office of Basic Energy Sciences and the National Science Foundation. The second author(K. Kang) was partially supported by NSF 
Grant No. DMS-9877055 and he thanks Professor B. Cockburn for helpful discussions. The authors are also grateful for the support of the Minnesota Supercomputer Institute and the Institute for Mathematics and its Applications.

\section{References}

[1] A. S. Almgren, J. B. Bell, And W. G. Szymczak, A numerical method for the incompressible Navier-Stokes equations based on an approximate projection, SIAM J. Sci. Comput. 17 (1996), no. 2, 358-369.

[2] Ann S. Almgren, John B. Bell, Phillip Colella, louis H. Howell, Michael L. Welcome, A Conservative Adaptive Projection Method for the Variable Density Incompressible Navier-Stokes Equations, J. Comput. Phys. 142 (1998), no. 1, 1-46

[3] D. Anderson and G.B. McFadden, A diffuse-interface description of internal waves in a near critical fluid, Phys. Fluids 9 (1997), 1870.

[4] D. Anderson, G.B. McFadden and A.A. Wheeler, Diffuse interface methods in fluid mechanics, Ann. Rev. Fluid Mech. 30, 139 (1998).

[5] J. W. BARReTt AND J. F. BLOWEy, Finite element approximation of a model for phase separation of a multi-component alloy with non-smooth free energy, Numer. Math. 77 (1997), no. 1, 1-34.

[6] J. W. Barrett And J. F. Blowey, Finite element approximation of the CahnHilliard equation with concentration dependent mobility, Math. Comp. 68 (1999), no. 226, 487-517.

[7] J. W. BARRett AND J. F. Blowey, An optimal error bound for a finite element approximation of a model for phase separation of a multi-component alloy with nonsmooth free energy, M2AN Math. Model. Numer. Anal. 33 (1999), no. 5, 971-987.

[8] J. W. Barrett, J. F. Blowey, AND H. Garcke, Finite element approximation of the Cahn-Hilliard equation with degenerate mobility SIAM J. Numer. Anal. 37 (1999), no. $1,286-318$.

[9] J. W. BarRett AND J. F. Blowey, Finite element approximation of an AllenCahn/Cahn-Hilliard system, IMA J. Numer. Anal. 22 (2002), no. 1, 11-71.

[10] P. W. BATES AND G. Fusco, Equilibria with many nuclei for the Cahn-Hilliard equation, J. Differential Equations 160 (2000), no. 2, pp. 283-356.

[11] A. BRANDT, Guide to multigrid development, in Multigrid Method, W. Hackbusch and U. Trottenberg, ed., Springer-Verlag, Berlin, 1982.

[12] D. Brown, R. Cortez and M. Minion, Accurate projection methods for the incompressible Navier-Stokes equations, J. Comp. Phys. 168 (2001), 168.

[13] J.W. Cahn, On spinodal decomposition, Acta Metall. 9 (1961), 795. 
[14] J. W. CAHN AND J. E. HILliARD, Free energy of a nonuniform system. I. Interfacial free energy, J. Chem. Phys. 28 (1958), 258-267.

[15] R. Chella and J. Vinals, Mixing of a two-phase fluid by cavity flow, Phys. Rev. E 53 (1996), 3832.

[16] M. COPETTI, Numerical experiments of phase separation in ternary mixtures, Math. Comput. Simulation 52 (2000), no. 1, pp. 41-51.

[17] M. Copetti AND C.M. Elliot, Kinetics of phase decomposition processes: numerical solutions to the Cahn-Hilliard equation, 52 (2000), no. 1, pp. 41-51.

[18] F. Dell'Isola, H. Gouin and G. Rotoli, "Radius and surface tension of microscopic bubbles by second gradient theory," Eur. J. Mech. B/Fluids 15, 545 (1996).

[19] F. Dell' Isola, H. Gouin and P. Seppecher, "Nucleation of spherical shell-like interfaces by second gradient theory: numerical simulations," C.R. Acad. Sci. Paris 320, 211 (1995).

[20] Q. DU AND R. NiCOlaides, Numerical analysis of a continuum model of phase transition, SIAM J. Num. Anal. 28 (1991), No. 5, pp. 1310-1322.

[21] C. Elliott, The Cahn-Hilliard model for the kinetics of phase separation, Mathematical Models for Phase Change Problems, J. Rodrigues ed., Int. Ser. Num. Mat. 88 pp. 35-73, Birkhäuser Basel 1989.

[22] C. Elliott And D. French, Numerical studies of the Cahn-Hilliard equation for phase separation, IMA J. Appl. Math. 38 (1987), pp. 97-128.

[23] C. M. Elliott AND D. A. FRench, A nonconforming finite-element method for the two-dimensional Cahn-Hilliard equation, SIAM J. Numer. Anal. 26 (1989), no. 4, 884-903.

[24] C. Elliott, D. French, And F. Milner, A second order splitting method for the Cahn-Hilliard equation, Numer. Math. 54 (1989), pp. 575-590.

[25] C. M. ElliotT AND STIG. LARSSON, Error estimates with smooth and nonsmooth data for a finite element method for the Cahn-Hilliard equation, Math. Comp. 58 (1992), no. 198, 603-630, S33-S36.

[26] D.J. EYRE, Systems for Cahn-Hilliard equations, SIAM J. Appl. Math. 53 (1993), pp. 1686-1712.

[27] D. A. French AND J. W. SchaefFer, Continuous finite element methods which preserve energy properties for nonlinear problems, Appl. Math. Comput. 39 (1990), no. 3, 271-295.

[28] D. FuRIHATA, Finite Difference Schemes for $\frac{\partial u}{\partial t}=\left(\frac{\partial}{\partial x}\right)^{\alpha} \frac{\delta G}{\delta u}$ that inherit energy conservation or dissipation property, J. Comp. Phys. 156 (1999), pp. 181-205.

[29] D. Furihata, Daisuke A stable and conservative finite difference scheme for the Cahn-Hilliard equation, Numer. Math. 87 (2001), no. 4, pp. 675-699. 
[30] M.E. Gurtin, D. Polignone and J. Vinals, Two-phase binary fluids and immiscible fluids described by an order parameter, Math. Models and Methods in Appl. Sci. 6, 815 (1996).

[31] J. Hageman, A study of pinchoff and reconnection of an unstably stratified fluid layer, M.S. Thesis, Department of Aerospace and Mechanics, University of Minnesota, 1999.

[32] P.C. Hohenberg and B.I. Halperin, Theory of dynamic critical phenomena, Rev. Mod. Phys. 49 (1977), 435.

[33] D. Jacqmin, Calculation of two-phase Navier-Stokes flows using phase-field modeling, J. Comp. Phys. 155 (1999), 96.

[34] D. Jacqmin, Contact line dynamics of a diffuse interface, J. Fluid Mech. 402 (2000), 57.

[35] D. Jasnow and J. Vinals, Coarse-grained description of thermocapillary flows, Phys. Fluids 8, 660 (1996).

[36] G.-S. JiAnG AND C.-W. SHU, Efficient implementation of weighted ENO schemes, J. Comput. Phys. 126 (1996), no. 1, 202-228.

[37] J.S. Kim and J. Lowengrub, On the pinchoff of liquid/liquid jets with surface tension, in preparation.

[38] J.S. Kim and J. Lowengrub, Diffuse interface modeling of three-fluid flows with surface tension, in preparation.

[39] D. KWAK, V-cycle multigrid for cell-centered finite differences, SIAM J. Sci. Comput., 21 (1999), no. 2, pp. 552-564.

[40] H.Y. Lee, J.S. Lowengrub And J. Goodman, Modeling pinchoff and reconnection in a Hele-Shaw cell. I. The models and their calibration, Phys. Fluids 14 (2002), no. 2, pp. $492-513$.

[41] H.Y. Lee, J.S. Lowengrub And J. Goodman, Modeling pinchoff and reconnection in a Hele-Shaw cell. II. Analysis and simulation in the nonlinear regime , Phys. Fluids 14 (2002), no. 2, pp. 514-545.

[42] E.K. Longmire, J.S. Lowengrb and D.L. Gefroh, A Comparison of Experiments and Simulations on Pinch-Off in Round Jets, in Proceedings of the 1999 ASME/JSME Meeting, San Francisco.

[43] J. Lowengrub, J. Goodman, H. Lee, E. Longmire, M. Shelley and L. Truskinovsky, Topological transitions in liquid/liquid interfaces, Chapman \& Hall/CRC Res. Notes Math, 409 (1999), 221.

[44] J. Lowengrub and L. Truskinovsky, Quasi-incompressible Cahn-Hilliard fluids and topological transitions., R. Soc. Lond. Proc. Ser. A Math. Phys. Eng. Sci. 454 no. 1978, (1998), 2617-2654. 
[45] Daniel F. Martin And Phillip Colella, A Cell-Centered Adaptive Projection Method for the Incompressible Euler Equations, J. Comput. Phys. 163 (2000), pp. 271-312.

[46] B. Nadiga and S. Zaleski, Investigations of a two-phase fluid model, Eur. J. Mech. B/Fluids 15, 885 (1996).

[47] A. Novick-Cohen And L. A. Peletier, Steady states of the one-dimensional Cahn-Hilliard equation, Proc. Roy. Soc. Edinburgh Sect.A 123 (1993), no. 6, pp. 1071-1098.

[48] I.C. Sanchez, Bulk and interface thermodynamics of polymer alloys, Ann. Rev. Mater. Sci. 13 (1983), 387.

[49] P. Seppecher, Moving contact lines in the Cahn-Hilliard theory, Int. J. Eng. Sci. 34 (1996), 977.

[50] L. de Sobrino, Note on capillary waves in the gradient theory of interfaces, Can. J. Phys. 63, 1132 (1985).

[51] L. de Sobrino and J. Peternelj, On capillary waves in the gradient theory of interfaces, Can. J. Phys. 63, 131 (1985).

[52] H. Struchtrup and J.W. Dold, Surface tension in a reactive binary mixture of incompressible fluids, IMA preprint 1708 (2000).

[53] Z. Z. Sun, A second-order accurate linearized difference scheme for the twodimensional Cahn-Hilliard equation, Math. Comp. 64 (1995), no. 212, pp 1463-1471.

[54] R. Temam, Infinite Dimensional Dynamical Systems in Mechanics and Physics, Springer-Verlag, New York, 1988.

[55] U. Trottenberg, C. Oosterlee, And A. Schüller, MUltiGrID,Academic press, 2001.

[56] M. Verschueren, A diffuse interface model for structure development in flow, Ph.D. Thesis, Technische Universiteit Eindhoven, the Netherlands, 1999.

[57] M. Vershueren, F.N. van de Vosse and H.E.H. Meijer, Diffuse-interface modeling of thermocapillary flow instabilities in a Hele-Shaw cell, J. Fluid Mech. 434 (2001), 153.

[58] S. ZHENG, Asymptotic behavior of the solution to the Cahn-Hilliard equation, Applic. Anal. 23 (1986), 165-184. 